Old Dominion University

ODU Digital Commons

Summer 2018

\title{
Novel Technique for Gait Analysis Using Two Waist Mounted Gyroscopes
}

Ahmed Nasr

Old Dominion University, ah.nasr@gmail.com

Follow this and additional works at: https://digitalcommons.odu.edu/computerscience_etds

Part of the Computer Sciences Commons

\section{Recommended Citation}

Nasr, Ahmed. "Novel Technique for Gait Analysis Using Two Waist Mounted Gyroscopes" (2018). Master of Science (MS), Thesis, Computer Science, Old Dominion University, DOI: 10.25777/xhmz-qy31

https://digitalcommons.odu.edu/computerscience_etds/41

This Thesis is brought to you for free and open access by the Computer Science at ODU Digital Commons. It has been accepted for inclusion in Computer Science Theses \& Dissertations by an authorized administrator of ODU Digital Commons. For more information, please contact digitalcommons@odu.edu. 


\title{
NOVEL TECHNIQUE FOR GAIT ANALYSIS USING TWO WAIST MOUNTED GYROSCOPES
}

by

\author{
Ahmed Nasr \\ B.S. May 2006, Arab Academy for Science and Technology, Egypt
}

A Thesis Submitted to the Faculty of

Old Dominion University in Partial Fulfillment of the

Requirements for the Degree of

MASTER OF SCIENCE

COMPUTER SCIENCE

OLD DOMINION UNIVERSITY

August 2018

\author{
Approved by: \\ Tamer Nadeem (Director) \\ Ravi Mukkamala (Co-Director) \\ Michele Weigle (Member)
}




\title{
ABSTRACT \\ NOVEL TECHNIQUE FOR GAIT ANALYSIS USING TWO WAIST MOUNTED GYROSCOPES
}

\author{
Ahmed Nasr \\ Old Dominion University, 2018 \\ Director: Dr. Tamer Nadeem \\ Co-Director: Dr. Ravi Mukkamala
}

Analysis of the human gait is used in many applications such as medicine, sports, and person identification. Several research studies focused on the use of MEMS inertial sensors for gait analysis and showed promising results. The miniaturization of these sensors and their wearability allowed the analysis of gait on a long term outside of the laboratory environment which can reveal more information about the person and introduced the use of gait analysis in new applications such as indoor localization.

Step detection and step length estimation are two basic and important gait analysis tasks. In fact, step detection is a prerequisite for the exploration of all other gait parameters. Researchers have proposed many methods for step detection, and their experiments results showed high accuracies that exceeded $99 \%$ in some cases. All of these methods rely on experimental thresholds selected based on a limited number of subjects and walking conditions. Selecting and verifying an optimal threshold is a difficult task since it can vary according to a lot of factors such as user, footwear, and the walking surface material. Also, most of these methods do not distinguish walking from other activities; they can only recognize motion state from idle state. Methods that can be used to distinguish walking from other activities are mainly machine learning methods that need training and complex data labeling. On the other hand, step length estimation methods used in the literature either need constant calibration for each user, rely on impractical sensor placement, or both.

In this thesis, we employ the human walking bipedal nature for gait analysis using two MEMS gyroscopes, one attached to each side of the lower waist. This setup allowed the step detection and discrimination from other non bipedal activities without the need for magnitude thresholds or training. We were also able to calculate the hip rotation angle in the sagittal plane which allowed us to estimate the step length. 
without needing for constants calibration. By mounting an accelerometer on the center of the back of the waist, we were able to develop a method to auto-calibrate the Weinberg method constant, which is one of the most accurate step length estimation methods, and increase its accuracy even more. 
Copyright, 2018, by Ahmed Nasr, All Rights Reserved. 


\section{ACKNOWLEDGEMENTS}

Firstly, I would like to express my special thanks to my advisor Dr. Nadeem for his continuous support of my M.Sc. study. I would like also to thank the rest of my thesis committee: Dr. Mukkamala, and Dr. Weigle, for their insightful comments and encouragement.

My sincere thanks to my friend and brother Dr. Ahmed Salem for his help and support.

To my wife and kids, thank you for everything. 


\section{TABLE OF CONTENTS}

LIST OF TABLES viii

LIST OF FIGURES

$\mathrm{x}$

CHAPTER

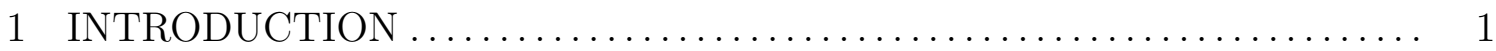

1.1 WHAT IS GAIT ANALYSIS $\ldots \ldots \ldots \ldots \ldots \ldots \ldots \ldots \ldots \ldots \ldots \ldots \ldots \ldots \ldots \ldots$

1.2 GAIT ANALYSIS USING INERTIAL SENSORS . . . . . . . . . . . 2

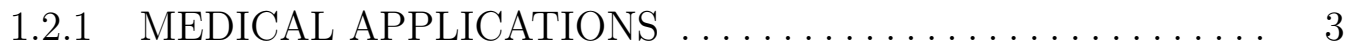

1.2 .2 INDOOR LOCALIZATION $\ldots \ldots \ldots \ldots \ldots \ldots \ldots \ldots \ldots \ldots \ldots$

1.2 .3 BIOMETRIC IDENTIFICATION . . . . . . . . . . . . . 4

1.3 ISSUES WITH INERTIAL SENSORS-BASED TECHNIQUES . . . . 4

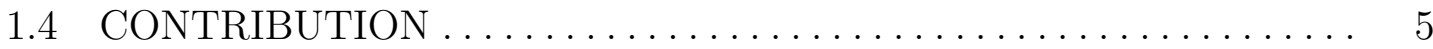

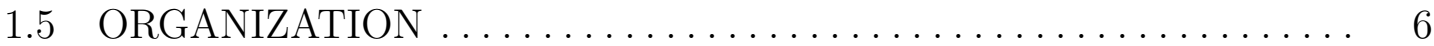

2 BACKGROUND AND RELATED WORK $\ldots \ldots \ldots \ldots \ldots \ldots \ldots \ldots \ldots \ldots$

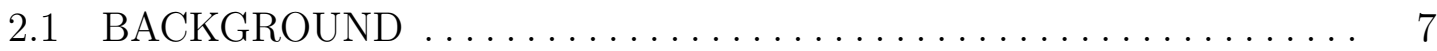

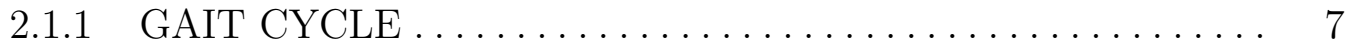

2.1.2 MEMS INERTIAL SENSORS $\ldots \ldots \ldots \ldots \ldots \ldots \ldots \ldots \ldots$

Drift Error $\ldots \ldots \ldots \ldots \ldots \ldots \ldots \ldots \ldots \ldots \ldots \ldots \ldots \ldots \ldots \ldots \ldots \ldots$

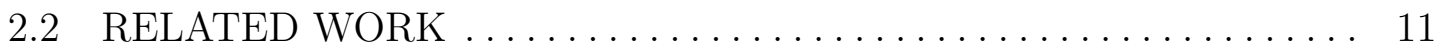

2.2.1 STEP DETECTION METHODS . . . . . . . . . . . . . . . 11

Basic Peak and Zero-Crossing Detection Techniques........ . 12

Stance Detection . . . . . . . . . . . . . . . . . . . . . . . 12

Dynamic Time Warping . . . . . . . . . . . . . . . . . . . 13

Normalized Auto-correlation based Step Counting (NASC) . . . 13

Machine Learning Techniques ..................... 14

2.2.2 STEP LENGTH ESTIMATION METHODS . . . . . . . . . . . . . . 14

Zero-velocity Updates $(Z U P T) \ldots \ldots \ldots \ldots \ldots \ldots \ldots \ldots \ldots$

Swing Angle ............................. 15

Center of Mass $(\mathrm{CoM})$ vertical acceleration . . . . . . . . . . 16

Modified Pendulum Model. . . . . . . . . . . . . . . . . . . . . 16

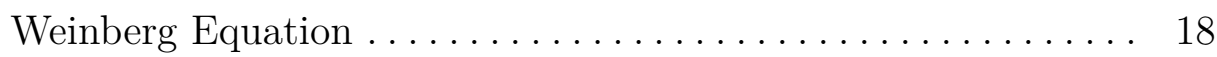

Step Frequency Model ....................... 18

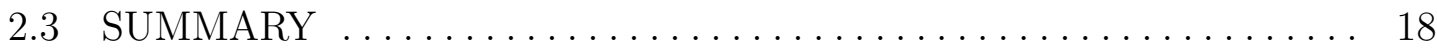


3 SYSTEM ARCHITECURE . . . . . . . . . . . . . . . . . . . . 20

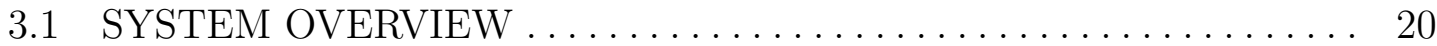

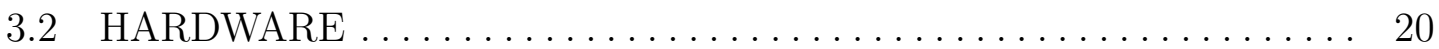

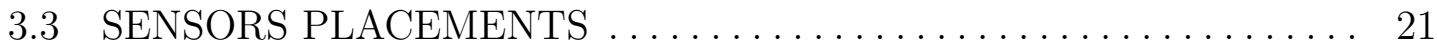

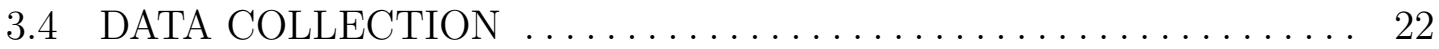

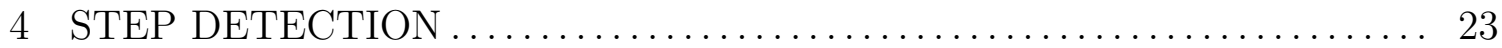

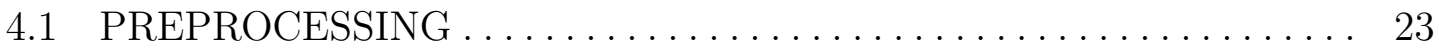

4.2 STEP DETECTION ALGORITHM $\ldots \ldots \ldots \ldots \ldots \ldots \ldots \ldots \ldots \ldots$

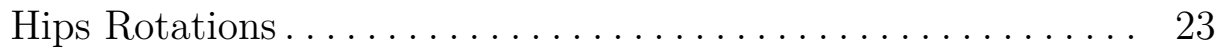

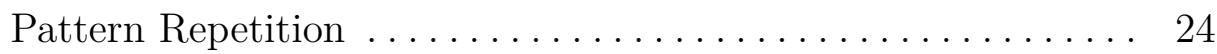

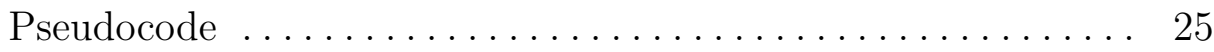

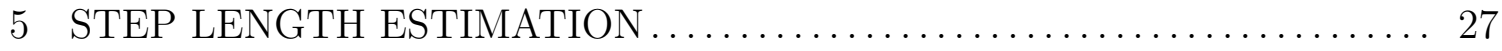

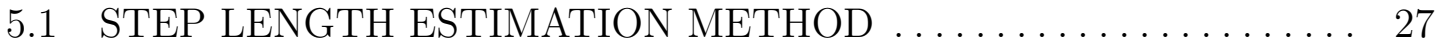

5.2 WEINBERG METHOD AUTO-CALIBRATION ............ 28

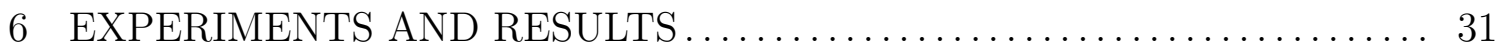

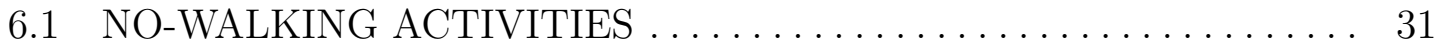

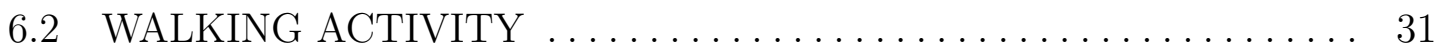

6.2 .1 STEP DETECTION RESULTS ................ 33

6.2.2 STEP LENGTH ESTIMATION RESULTS . . . . . . . . . . . . . 34

7 CONCLUSION AND FUTURE WORK $\ldots \ldots \ldots \ldots \ldots \ldots \ldots \ldots \ldots \ldots \ldots \ldots$

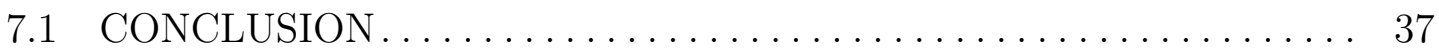

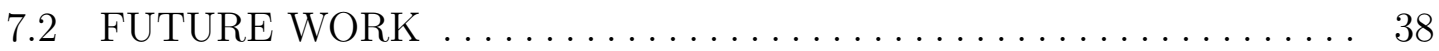

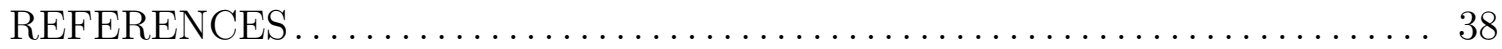

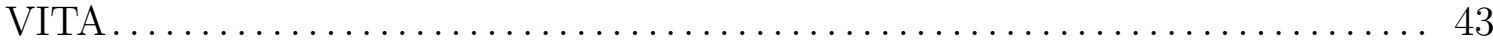




\section{LIST OF TABLES}

Table

Page

$1 \quad$ Gait Parameters ............................... 1

2 Step Detection and Step Length Estimation Related Parameters . . . . . . . 2

3 Slow Gait Speed and Step Frequency .................. 33

$4 \quad$ Regular Gait Speed and Step Frequency . . . . . . . . . . . . . . . . . 33

$5 \quad$ Fast Gait Speed and Step Frequency $\ldots \ldots \ldots \ldots \ldots \ldots \ldots \ldots \ldots \ldots \ldots$

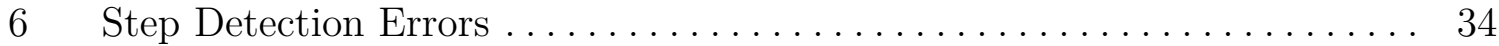

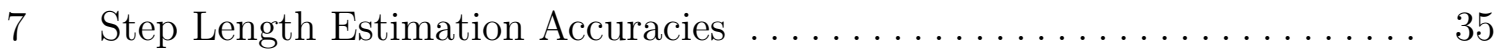




\section{LIST OF FIGURES}

Figure Page

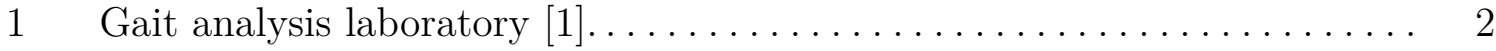

2 Common inertial sensors placements for gait analysis $[2] \ldots \ldots \ldots \ldots \ldots$

3 Our Gait Analysis System Objectives. .................... 5

4 The gait cycle with reference to the right foot, where HS is the heel strike, CTO is the contralateral toe off the ground, CHS is the contralateral heel strike, and TO is the toe off the ground $[3] \ldots \ldots \ldots \ldots \ldots \ldots \ldots$

$5 \quad$ Walking inverted pendulum model $\ldots \ldots \ldots \ldots \ldots \ldots \ldots \ldots$

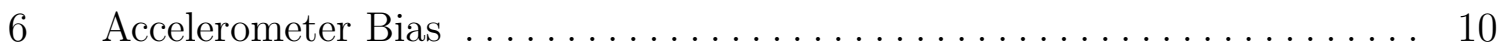

$7 \quad$ Step length estimation using a single and double support model $[15] \ldots \ldots$

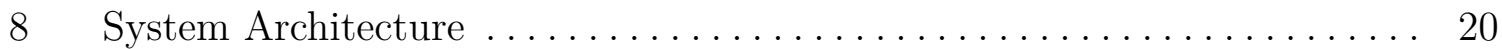

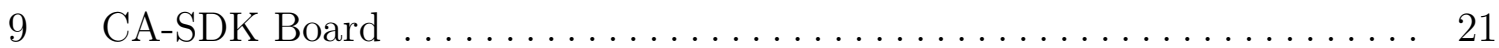

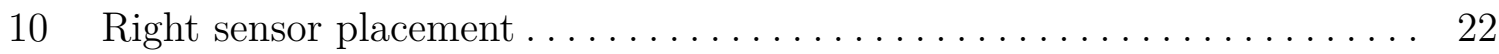

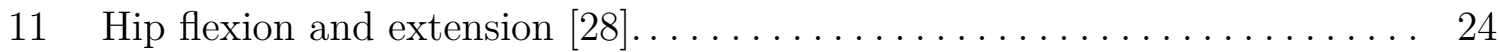

12 Plot of two waist mounted gyroscopes filtered data during walking. $\Delta t_{1}$ is the time between the first step and the second step, and $\Delta t_{2}$ is the time

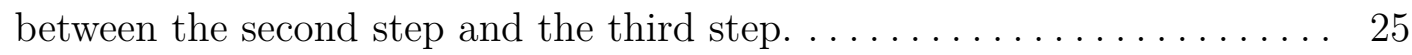

13 Walking inverted pendulum model angles $\ldots \ldots \ldots \ldots \ldots \ldots \ldots$

14 Plot of two waist mounted gyroscopes filtered data during no-walking activities. A1 corresponds to the activity of rocking on a chair, A2 to twirling on a chair, A3 to transitioning between standing and sitting and A4 jumping in place.

15 Plot of two waist mounted gyroscopes filtered data during walking at

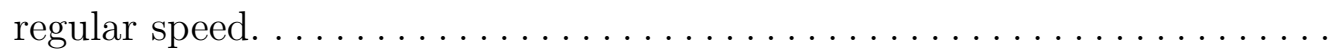

16 Plot of the step lengths estimations as a percentage of the real steps

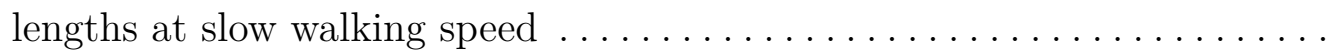


17 Plot of the step lengths estimations as a percentage of the real steps

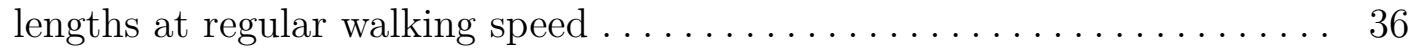

18 Plot of the step lengths estimations as a percentage of the real steps

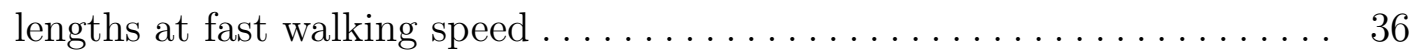




\section{CHAPTER 1}

\section{INTRODUCTION}

\subsection{WHAT IS GAIT ANALYSIS}

Gait analysis is the identification, measurement and evaluation of walking related parameters. These parameters are categorized into kinetics, kinematics, spatial, and temporal parameters. Table 1 shows some gait parameters examples that fall under each category. The analysis of the human gait can be used in a broad range of applications including sports, medical, and person identification applications.

\section{TABLE 1: Gait Parameters}

\begin{tabular}{|c|c|c|c|}
\hline Kinetic & Kinematic & Spatial & Temporal \\
\hline Joints forces & Joints angles & Step length & Cadence \\
Joints torques & Joints velocities & Stride length & Speed \\
& Joints accelerations & Step width & Step duration \\
& & & Stride duration \\
\hline
\end{tabular}

Step detection and step length estimation are two of the most important tasks of gait analysis. In fact, step detection is a prerequisite for the analysis of the other gait parameters including step length. The step length is the output of the complex activities of the joints and muscles during walking, so it is related in some way to the other gait parameters. Table 2 lists some of the gait parameters that can be directly estimated based on the detection and length estimation of the step. The vertical displacement of the center of mass (CoM) and the hip flexion which is the rotation of the hip in the direction of walking can be estimated based on the step length if the leg length is known. In this thesis, we will focus on the step detection and step length estimation as the main parameters of gait analysis.

Several methods can be used for gait analysis including clinical examination by a specialist, image processing, and floor sensors [18]. Figure 1 shows a gait analysis laboratory equipped with floor sensors and cameras. These methods need special setup 
TABLE 2: Step Detection and Step Length Estimation Related Parameters

\begin{tabular}{|c|c|}
\hline Step Detection & Step Length \\
\hline Cadence & Step Speed \\
Step Time & CoM Vertical Displacement \\
Stride Time & Hip Flexion \\
\hline
\end{tabular}

and cannot be used for a long-term analysis. Another method that does not suffer from these problems is the analysis of gait using wearable systems that uses sensors placed on the body. Wearable systems have brought the convenience of real-time gait analysis outside of laboratory conditions for a long-term which can provide more useful information about persons' daily life and enabled the use of gait analysis in new applications such as indoor localization. Wearable systems can still be used in laboratories as well, with the advantage of a lower cost analysis than the methods that require special setup.

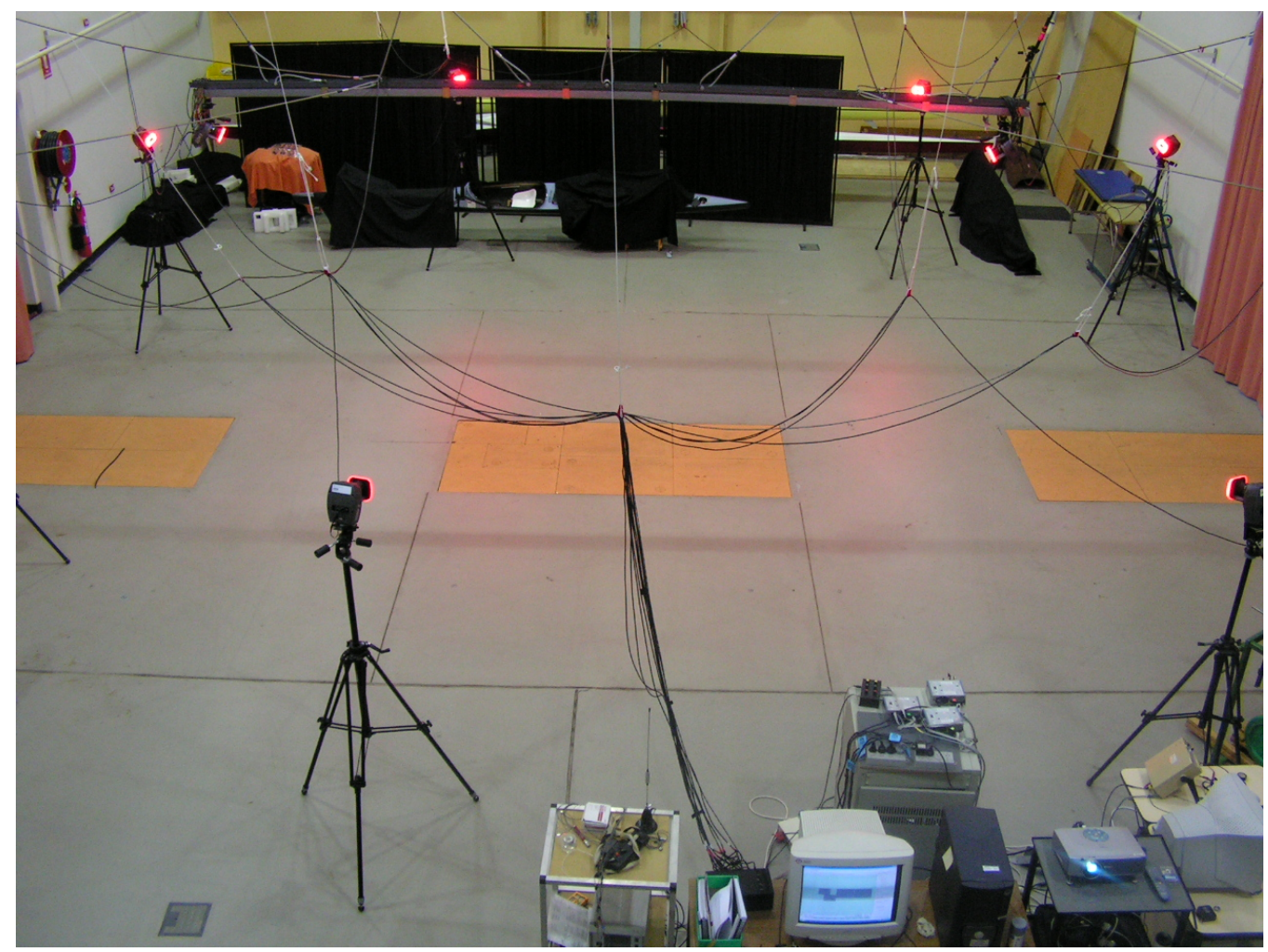

FIG. 1: Gait analysis laboratory [1]. 


\subsection{GAIT ANALYSIS USING INERTIAL SENSORS}

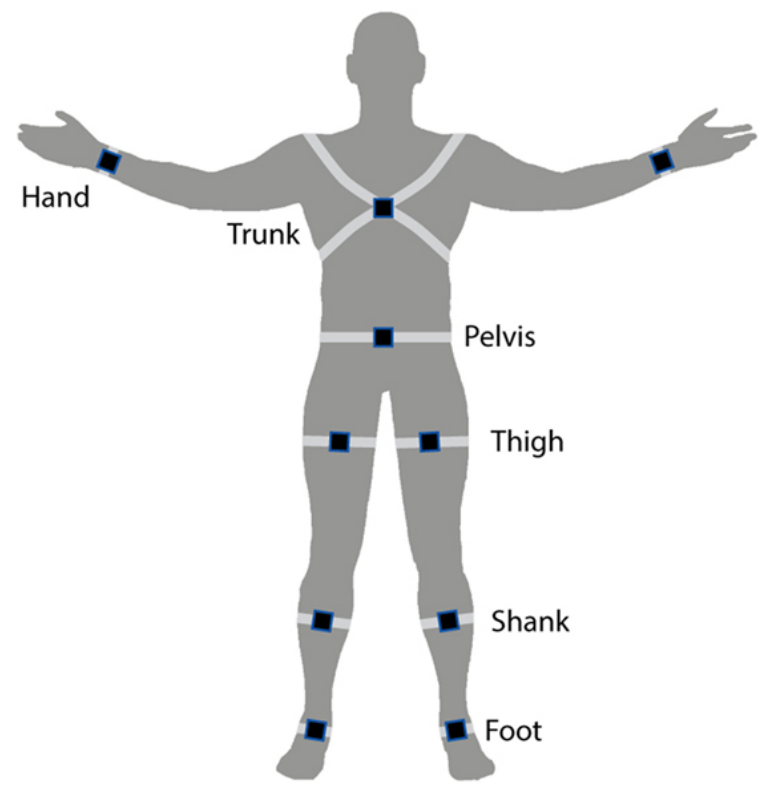

FIG. 2: Common inertial sensors placements for gait analysis [2].

Microelectromechanical Systems (MEMS) technology enabled the miniaturization of inertial sensors including accelerometers and gyroscopes so they can be used in wearable systems. An accelerometer is a device used to measure linear acceleration and a gyroscope is a device used to measure angular velocity. MEMS inertial sensors became widely used in wearable systems for gait analysis due to their small size, light weight, low cost, and low power consumption. Figure 2 shows MEMS inertial sensors common placements for gait analysis. We highlight the use of MEMS inertial sensors for gait analysis in medical applications, indoor localization, and biometric identification.

\subsubsection{MEDICAL APPLICATIONS}

An example that shows the advantage of using MEMS inertial sensors for gait analysis in medical applications is the long-term gait monitoring of Parkinson Disease symptoms in [6]. An accelerometer and a gyroscope mounted on the shank were used to monitor five patients and results showed special characteristics of Parkinsonian gait and that long-term changes in stride length can be used to estimate the fluctuation of efficacy of Levodopa therapy used to manage the motor symptoms of Parkinson Disease. 


\subsubsection{INDOOR LOCALIZATION}

Indoor localization is the problem of locating persons in places where the Global Positioning System (GPS) cannot be used. Using inertial sensors for step detection, step length and heading estimation enabled indoor localization systems that do not need deployment of special-purpose infrastructure or calibration with an existing

infrastructure. In [4], the inertial sensors of a smartphone, a WiFi scanner and a map were used by a particle filter for indoor localization. Other systems were built without the need of existing infrastructure help using inertial sensors mounted on the foot [20] and of a smartphone [5].

\subsubsection{BIOMETRIC IDENTIFICATION}

Gait recognition has been proposed as a biometric feature. By using a 3-axis accelerometer and a 2-axis gyroscope mounted on the leg, a method was proposed to identify people from their gait in [19]. The method is based on the segmentation of gait into cycles, which is essentially a stride detection method. The accuracy of the system achieved $100 \%$ for 4 subjects, which is very promising and encourages more research in this area.

\subsection{ISSUES WITH INERTIAL SENSORS-BASED TECHNIQUES}

Researchers have proposed many methods for step detection and their experiments results showed high accuracies that exceeded $99 \%$ in some cases [4]. All of these methods rely on experimental thresholds selected based on a limited number of subjects and walking conditions. Selecting and verifying an optimal threshold is a difficult task since it will vary according to a lot of factors such as users, footwear and the walking surface material [7]. Also, most of these methods do not distinguish walking from other activities, they can only recognize motion state from idle state [8]. The methods that can be used to distinguish walking from other activities, such as in [9], are based on machine learning techniques that need training and complex data labeling. On the other hand, step length estimation methods used in the literature either need calibration of constants for each user, rely on impractical sensor placement or both. 


\subsection{CONTRIBUTION}

In this thesis, we use two gyroscopes mounted on the lower left and right sides of the waist instead of a single inertial sensor for step detection and step length estimation. One gyroscope is mounted as close as possible to the right upper hip bone while the other to the left upper hip bone. This setup provides data about the angular motion of the hips during walking and a fixed pattern between the two of them that can exploited for step detection. We use this pattern to distinguish walking from other non-bipedal activities and reduce the rate of false steps detected without using magnitude thresholds.

Using this setup, we also estimate the hip rotation angle in the sagittal plane and use it to estimate the step length using only the effective leg length without using constants that need to be calibrated. We use also the hip rotation angle with the vertical displacement of the body during walking to auto-calibrate the constant of the Weinberg step length estimation method which increases its accuracy and practicality. We estimated the body center of mass vertical displacement during walking using a classical method by placing an accelerometer on the back center of the waist.

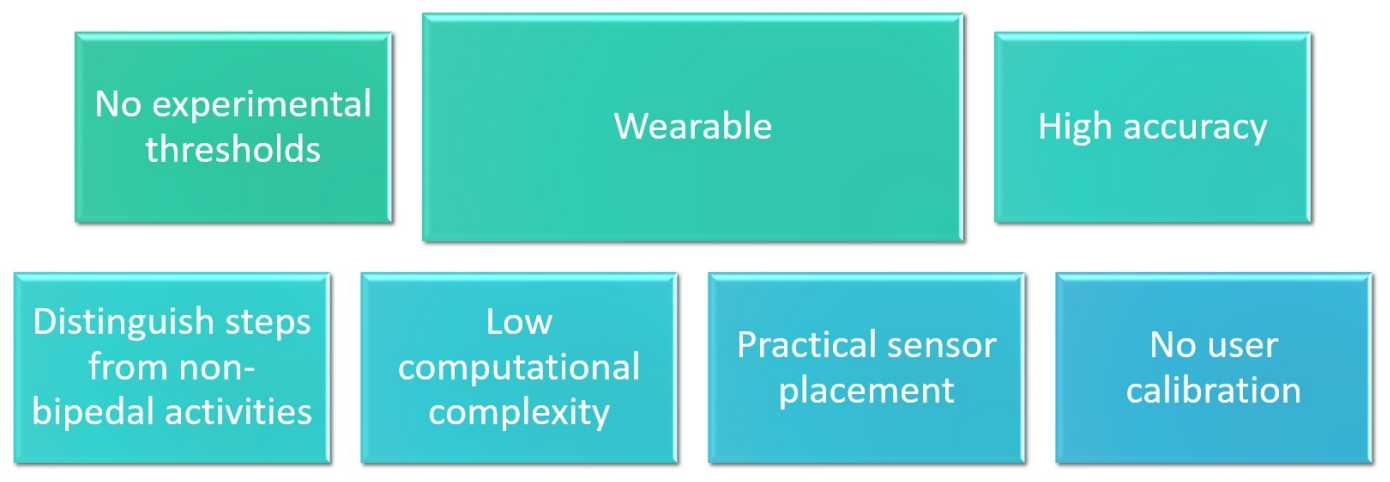

FIG. 3: Our Gait Analysis System Objectives.

Our objective was to build an accurate gait analysis system that is easy and practical to wear to allow long term monitoring without affecting the user experience. The accuracy of our system is increased by distinguishing walking steps from non-bipedal activities to reduce the false positives rate. The elimination of user calibration will increase the system accuracy as the calibration is user prone, and it will also increase the user friendliness. Our system is not based on any experimental 
magnitude or similarity distance thresholds, so it can be easily implemented and can be used by different users in different walking conditions. Our system requirements are summarized in Figure 3.

\subsection{ORGANIZATION}

We present some of the step detection and step length estimations methods used in the literature in Chapter 2. In Chapter 3, we give an overview of our gait analysis system, describe the hardware used, and the data collection method. We present our novel step detection method in Chapter 4, and our novel step length estimation and Weinberg auto-calibration methods in Chapter 5. We evaluate the performance of the system with different subjects and walking speeds and we compare our step length estimation and the auto-calibration method accuracies to two other step length estimation techniques in Chapter 6. Then we conclude with Chapter 7. 


\section{CHAPTER 2}

\section{BACKGROUND AND RELATED WORK}

\subsection{BACKGROUND}

\subsubsection{GAIT CYCLE}

The gait cycle can be defined as the period between two subsequent ground contacts of the same foot as illustrated in Figure 4. The step length is the distance between the ground contact point of one foot and the successive ground contact point of the other foot. The stride length is the distance between successive ground contact points of the same foot which is the step length multiplied by 2 .

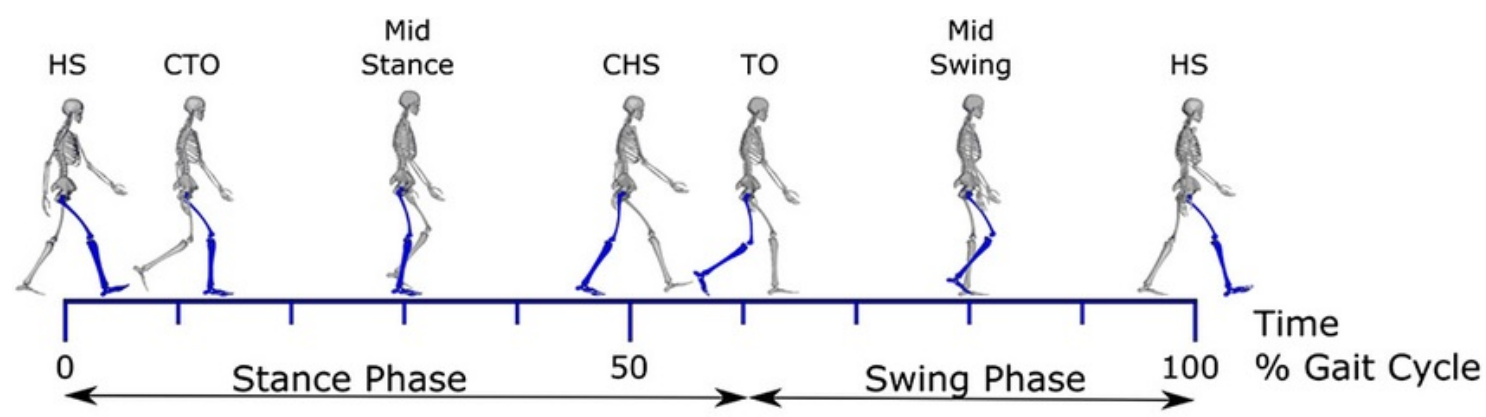

FIG. 4: The gait cycle with reference to the right foot, where HS is the heel strike, CTO is the contralateral toe off the ground, CHS is the contralateral heel strike, and TO is the toe off the ground [3].

For each leg, the gait cycle can be divided into 2 phases, the stance phase where the foot is in contact with the ground and the swing phase where the foot is not in contact with the ground. During walking, at least one foot has to be in the stance phase. The period where only one foot is in contact with the ground is called the single support while the double support is the period where both feet are in contact with the ground.

The stance phase involves a series of important events, the heel strike which is the initial foot contact with the ground, foot flat, mid-stance, heel off and toe off (final 


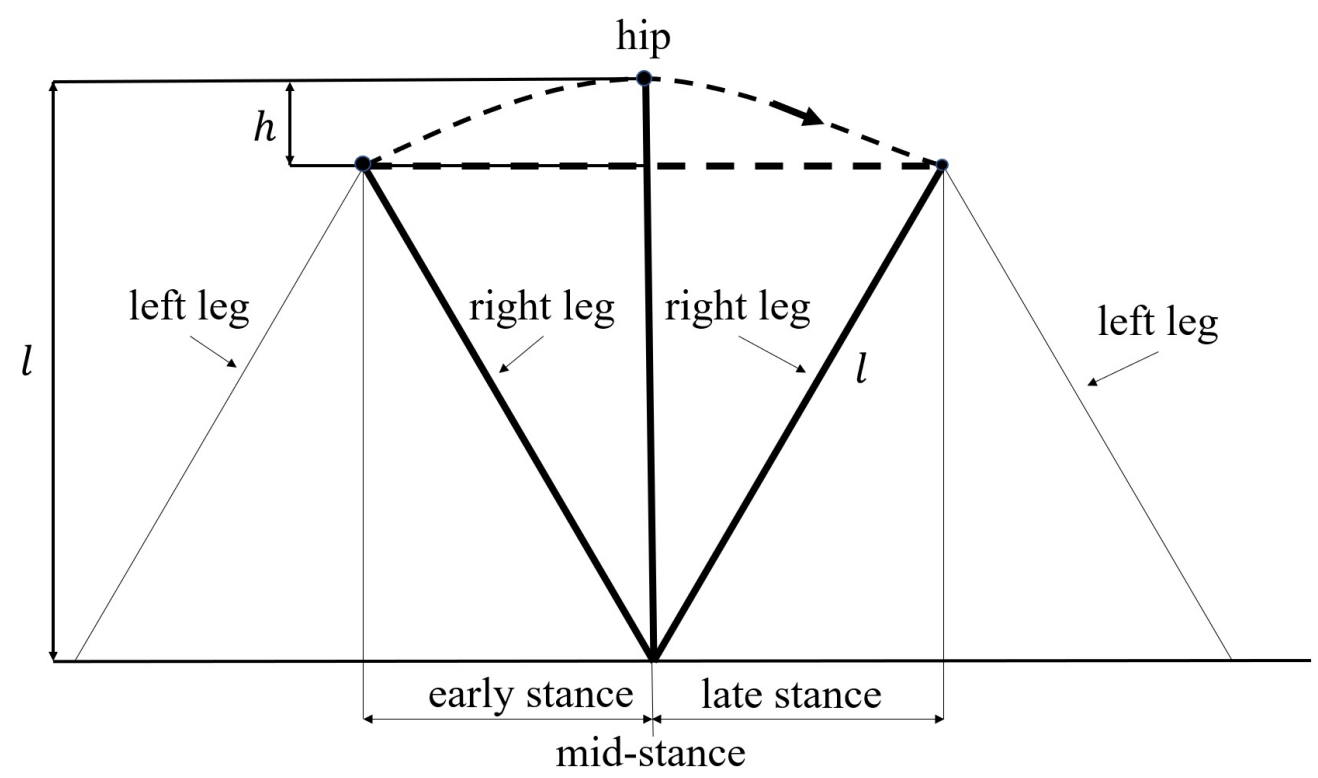

FIG. 5: Walking inverted pendulum model

contact). The mid-stance is the event of the body weight passing over the stationary foot, it occurs at the same time of the swing leg's mid-swing event. We can refer to the interval between heel strike and mid-stance by early stance, and the interval between mid-stance and toe-off by late stance.

To simplify and understand human walking, a simple model of an inverted pendulum is used for the stance phase where the upper body is represented as a point of mass at the hip rotating over the foot in contact with the ground [17]. This model suggests that the body moves forward during walking following a compass path. The stance phase inverted pendulum model is illustrated in Figure 5 where $l$ is the leg length from the hip to the ground representing the pendulum length and $h$ is the distance of hip displacement resulted from the gait compass path.

\subsubsection{MEMS INERTIAL SENSORS}

Many gait parameters can be analyzed using accelerometers and gyroscopes due to their ability to detect and measure different aspects of motion. Accelerations, velocities and displacements can be measured using accelerometers by sensing the body part acceleration and then velocity and displacement can be obtained by the mean of the 1st and 2nd integration. Gyroscopes can measure joint rotations by 
sensing the angular velocity which can be integrated to get the angle of rotation. MEMS technology has made this feasible since it allowed the miniaturization of these sensors so they can be easily mounted on different body parts, but this came at the cost of accuracy as MEMS inertial sensors suffer from significant and different types of errors.

The most significant type of error that affects MEMS inertial sensors is the bias. The bias can be described as the offset of the output signal from the true signal, and it can be calculated by taking the average of the sensor's output signal in an idle state. Figure 6 shows an accelerometer signal and its bias when no movement is applied to the sensor.

\section{Drift Error}

To understand the effect of the bias on the integration of the gyroscope's data, assume that the true angular velocity is $\omega$, the angular velocity measured by the gyroscope is $\hat{\omega}$ and the bias is $b_{\omega}$, then the gyroscope's output angular velocity is given by the following equation:

$$
\hat{\omega}=\omega+b_{\omega}
$$

To get the angle of rotation $\hat{\theta}$, the angular velocity $\hat{\omega}$ will be integrated, and $\hat{\theta}$ will be given according to the following equations:

$$
\begin{gathered}
\hat{\theta}=\int \omega+b_{\omega} d t \\
\hat{\theta}=\theta+b_{\omega} \Delta t
\end{gathered}
$$

where $\theta$ is the true angle of rotation and $\Delta t$ is the time interval of the integration. The bias error grows with time when trying to calculate the angle of rotation using direct integration of the angular velocity causing a linear drift from the true angle.

The situation gets even worse when trying to calculate the displacement using double integration of the accelerometer data. Assume that the true acceleration is $a$, the measured acceleration is $\hat{a}$ and the accelerometer bias is $b_{a}$. The displacement $\hat{d}$ calculated using double integration of the accelerometer data is given by:

$$
\begin{gathered}
\hat{a}=a+b_{a} \\
\hat{v}=v+b_{a} \Delta t
\end{gathered}
$$




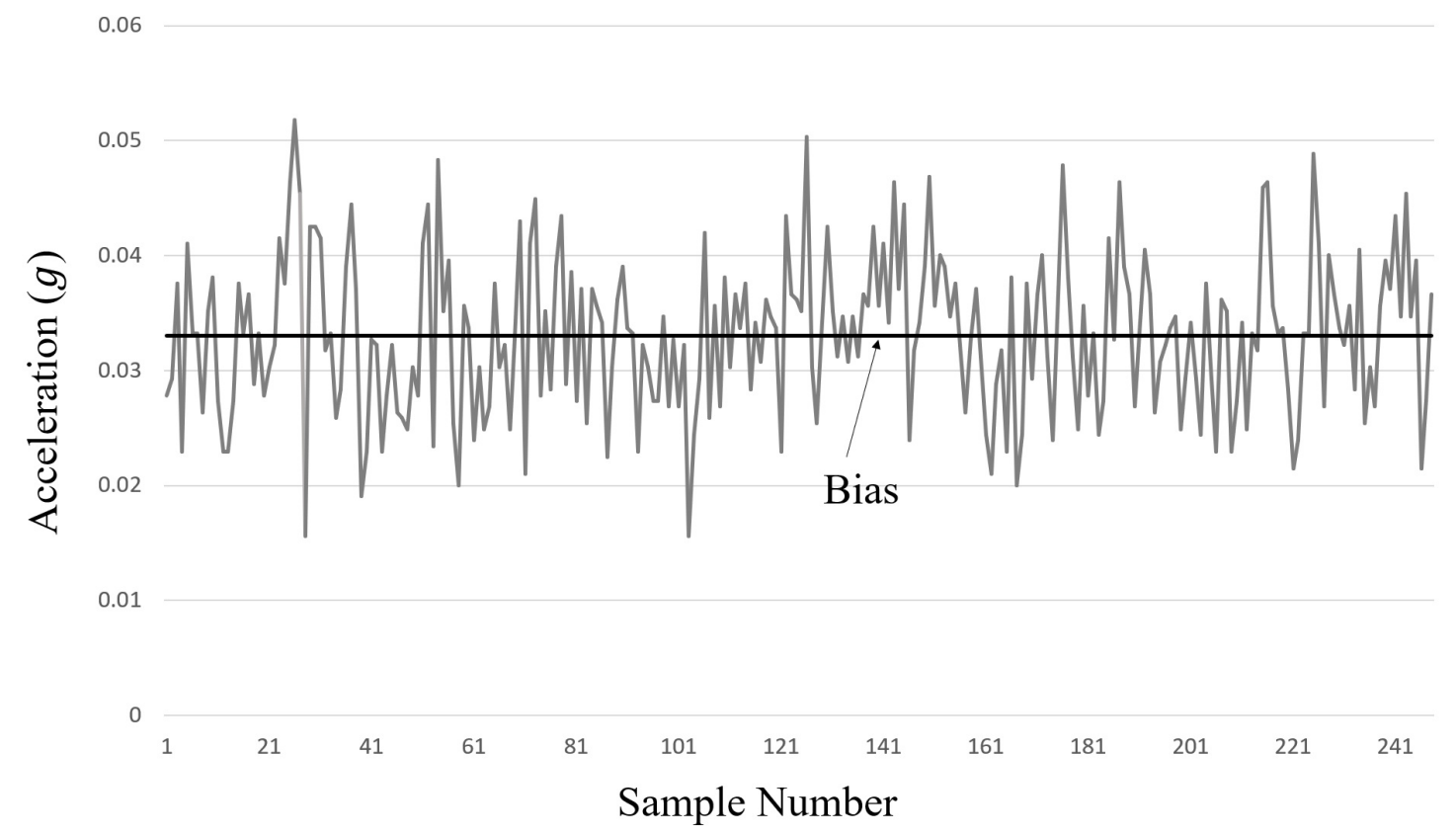

FIG. 6: Accelerometer Bias

Accelerometer signal and its bias in idle state. The acceleration is measured in terms of the gravity acceleration $g$. The bias is calculated by taking the average of the data.

$$
\hat{d}=d+b_{a} \Delta^{2} t
$$

where $v$ is the true velocity, $\hat{v}$ is the calculated velocity using first integration, $d$ is the true displacement and $\Delta t$ is the time interval of the integration. The velocity error grows linearly in time and the displacement error grows with the square of time causing a quadratic drift from the true displacement.

One way to reduce the bias error is to calculate it in idle state then subtract it from the data afterwards. Unfortunately, this is not an easy task since the bias can change over time due to flicker noise and temperature [12]. The calculation of the accelerometer's bias is not an easy task as well since the accelerometer is affected by the gravity acceleration which will be present in idle state, so the exact orientation of the sensor has to be known in order not to calculate the gravity acceleration component as a bias.

Several methods can be used to reduce the inertial sensors drift such as sensor fusion of the accelerometer and the gyroscope using techniques such as Kalman filter, fusion GPS or magnetometers, and domain specific assumptions that allow the 
correction of data at instances of time where the data values are known [12].

\subsection{RELATED WORK}

Before estimating the step length, we need first to detect the step itself, but even though step detection is a prerequisite, the step length estimation is independent of the step detection algorithm and can be extended to search for any required information for the length estimation. In this section we begin by reviewing some of the step detection methods, then we review some of the step length estimation methods. Any step detection method can be paired with any step length estimation method as a part of a complete system.

\subsubsection{STEP DETECTION METHODS}

All of the step detection techniques that we have found in the literature use thresholds selected based on limited datasets and walking conditions to eliminate false positives. These thresholds can be magnitude and/or distance thresholds used to measure similarity between waveforms. The selection of these thresholds is experimental and it is hard to find a universal optimal threshold that fits different users, shoes, and walking surfaces. Some thresholding techniques are not even suitable for different walking speeds, so one will be forced to choose a high threshold leading to missing low speed steps or a low threshold leading to the detection of false steps [8].

Magnitude thresholds are used mainly to reject false steps that can result because of noise or some step events that can cause the same step to be counted more than once, they cannot be used to distinguish walking steps from other activities. They are often combined with other walking characteristics such as steps frequency range to reduce the number of false steps detected, but these selected characteristics often span many other activities as well.

Fitbit is an example of commercial step counting devices. Fitbit application allows the user to remove false steps detected by the Fitbit device. Fitbit devices will also miss steps that do not trigger the thresholds because of walking conditions such as walking on a very soft surface [10]. 


\section{Basic Peak and Zero-Crossing Detection Techniques}

The simplest step detection technique is to identify peaks in the accelerometer signal as steps if their values are greater than a threshold. This idea is based on that the up and down bounce of the body at each step will cause these peaks to happen. This technique is used in [22] using the magnitude of 3-axis accelerometer $\left(a_{3 D}\right)$ mounted on the waist.

$$
a_{3 D}=\sqrt{a_{x}^{2}+a_{y}^{2}+a_{z}^{2}}
$$

where $a_{x}, a_{y}$ and $a_{z}$ are the 3-axis accelerometer. A moving average filter is used to reduce noise in $a_{3 D}$, then a zero-crossing and peak detector are used. If a peak which is greater than a threshold and zero-crossing are sequentially detected, a step is generated by the detector.

A variation of this technique was used in [11] using the gyroscope of a smart phone placed in the trouser pocket in the vertical position. The gyroscope $\mathrm{X}$ axis data is first filtered using a 6th order Butterworth low-pass filter with a cutoff frequency of $3 \mathrm{~Hz}$ since the mean speed of a fast gait is in the range of 2.5 steps per second. The strides are detected by searching for two points zero-crossing one positive and the other is negative. To avoid false detection that can happens due to the fluctuation of the signal around the x-axis, a $100 \mathrm{~ms}$ timeout is used for the detector since two steps cannot occur within this time frame. A calibration is required where the user walk at a low speed to identify a minimum threshold. A manual correction was used to eliminate the false strides detected before placing the phone in the pocket.

\section{Stance Detection}

Recall that each gait cycle or stride consists of a swing phase and stance phase. During the stance phase the linear and angular velocities of the foot are supposed to be 0 . This idea was exploited in [23] using a gyroscope mounted on the foot. The total angular velocity $\omega_{\text {total }}$ of the gyroscope is first calculated using the following equation:

$$
\omega_{\text {total }}=\sqrt{\omega_{x}^{2}+\omega_{y}^{2}+\omega_{z}^{2}}
$$

where $\omega_{x}, \omega_{y}$ and $\omega_{z}$ are the gyroscope values of the 3 sensor frame's axis. Since $\omega_{\text {total }}$ will not be exactly equal to zero, it is compared to a threshold. Intervals with values greater than the threshold are considered to be the swing phases, while others with values less than the threshold are considered stance phases. 


\section{Dynamic Time Warping}

To minimize the false steps detected by the peak detection technique, a validation using Dynamic Time Warping (DTW) was used in [5]. The magnitude of the 3axis accelerometer of a smartphone is filtered using a $3 \mathrm{~Hz}$ low-pass Finite Impulse Response (FIR) filter, then the smoothed signal is searched for peaks and valleys to detect the steps. To reject peaks and valleys that do not represent a step, a magnitude threshold $\Delta_{a}$ and a time threshold $\Delta_{t}$ were used. To validate the detected steps and based on the expectation of steps similarity during a single gait, DTW was used. Suppose a candidate step $S_{i}$ is detected using the peak detection method, if the DTW distance between $S_{i}$ and $S_{i-2}$ is less than a threshold, both of them are considered real steps, otherwise $S_{i}$ will have another validation chance against $S_{i+2}$.

The reported error of this method is $1.1 \%$ with the smartphone placed in the pocket. This method's problem is that a tight distance threshold will cause false negatives in gaits with changes in walking speed, and a loose one will increase the false positives in general. Repetitive activities that cause cycles within the walking frequency in the accelerometer signal will be falsely detected as steps.

\section{Normalized Auto-correlation based Step Counting (NASC)}

In [4], the standard deviation of the accelerometer's data is calculated and compared to a threshold to discriminate between idle and motion states, if the standard deviation exceeded the threshold, the next processing step is applied to ascertain that the user is walking. Based on the repetitive nature of walking, the second processing step is to compute the auto-correlation of the accelerometer signal for a lag $\tau$. Since the step duration is not known, the algorithm tries values between predefined values $\tau_{\min }$ and $\tau_{\max }$. If the maximum auto-correlation value exceeded a threshold, the user is considered walking and $\tau_{\text {opt }}$ is set to the corresponding lag. The values of $\tau_{\min }$ and $\tau_{\max }$ are then set to $\tau_{\text {opt }}-10$ and $\tau_{\text {opt }}+10$. The final step of the algorithm generates a step for each $\frac{\tau_{o p t}}{2}$ of the walking period. This algorithm is independent of the sensor placement.

The reported error of this method was $0.6 \%$. However, another study shows that this method can detect transitioning between standing and sitting, and twirling on a chair as walking steps [8]. 


\section{Machine Learning Techniques}

A problem with the mentioned techniques is that they can be triggered by actions other than walking, jogging, and running. The DTW and NASC techniques are expected perform better than traditional peak and zero-crossing detection techniques regarding this issue but can still be triggered by any repetitive motion having the same periodicity of walking [8]. This can be avoided by using machine learning techniques such as the Adaptive Boosting (AdaBoost) technique used in [9]. The algorithm is based on waist-mounted accelerometer, gyroscope and magnetometer as well as other sensors. The sensors' data are processed to get the world 3-axis accelerations, angular rates, gravitational vector and frequency domain features of the acceleration and angular rates. The weak classifiers are chosen based on 12 different thresholds. The algorithm distinguish between forward, backward, sides walking, sitting and standing from a chair, going upstairs and downstairs, bending over, and no motion.

This method requires training and complex data labeling. The reported false positive error was $0.6 \%$, but the false negative error was $7.7 \%$ which is higher than the other methods.

\subsubsection{STEP LENGTH ESTIMATION METHODS}

One method for step length estimation is to assume a fixed length. The problem with this approach is apparent since different people have different step lengths even when walking at the same speed. This difference can reach $40 \%$ at the same speed [16]. Since the step length depends mainly on the person's leg length, we can be tempted to use a fixed step length for each person. However, the step lengths at different speeds for each person can vary by up to $50 \%$, so this method will also lead to a high error rate.

A better way to calculate the step length is to use wearable inertial sensors to estimate the lengths while the person is walking. Using direct double integration of the forward accelerometer then dividing it by the number of steps cannot be used since the bias error will grow significantly with time causing a quadratic drift as described before. Recall that one way to avoid this error is to use domain specific knowledge. Walking biomechanics can provide such knowledge for the step length estimation. 


\section{Zero-velocity Updates (ZUPT)}

In the stance phase, the linear velocity of the foot is known to be 0 . ZUPT uses this information to correct the velocities obtained from the 1st integration of the accelerometer data during the swing phase and to restart the integration at each swing phase, since the initial velocity is known in priori and hence stops the error propagation between steps. A detailed explanation of this method using a 3-axis accelerometer and gyroscope is found in [13]. The algorithm can be summarized by the following steps:

1. Integrate the gyroscope data to build the rotation matrix.

2. Transform the accelerometer data from sensor frame to world frame using the rotation matrix.

3. Integrate the transformed accelerations to get the linear velocities.

4. Calculate the mean velocity at the stance phase to estimate the integration errors accumulation.

5. Estimate the error in the velocities of the swing phase using linear interpolation of the stance phase mean velocity, then subtract the estimated errors from the velocities.

6. Integrate the corrected velocities to get the stride length.

The main advantages of this method are that it does not need user calibration or information, and it is always suitable to calculate running stride length. The main disadvantages are that the sensor placement is not practical and there is a need for extensive computations.

\section{Swing Angle}

In [6], a gyroscope was mounted around the shank of the leg and the swing leg angle $\theta$ was obtained by direct integration of the gyroscope data in the sagittal plane for each step. The estimated step length $S L$ was given by:

$$
S L=2 \sin \left(\frac{\theta}{2}\right)
$$

This method underestimates the step length since it does not take into account the body movement over the stance foot. To compensate for this underestimation, $S L$ 
was calibrated based on the relationship between the calculated step lengths and the true step lengths using a least-squares fit.

\section{Center of Mass (CoM) vertical acceleration}

Mounting the sensor on the waist is more practical and less intrusive than the foot and the shank, but unlike the foot, there are no periods of 0 velocity for the forward movement, so ZUPT cannot be applied to its forward acceleration.

Walking steps can be modeled as an inverted pendulum as illustrated in Figure 5 , where the body rotates over the foot in contact with the ground [27]. The authors of [14] have estimated the vertical displacement $h$ of the trunk resulting from the walking inverted pendulum model by double integrating the data of an accelerometer placed on the dorsal side of the trunk near the body Center of Mass (CoM). To avoid integration drift, a $0.1 \mathrm{~Hz}$ 4th order zero lag Butterworth high-pass filter was used. The step length $S L$ was then calculated using the following geometric relation:

$$
\begin{gathered}
S L=2 \sqrt{l^{2}-(l-h)^{2}} \\
S L=2 \sqrt{2 l h-h^{2}}
\end{gathered}
$$

where $l$ is the effective leg length measured from the sensor to the ground. This method underestimates the step length and has to be calibrated by multiplying the equation by a constant calculated for each user.

\section{Modified Pendulum Model}

An extension of the step length estimation model based on the CoM vertical was used in [15]. The new model takes into account the forward displacement of the trunk during the double support phase $L_{d s}$ from $t_{a}$ to $t_{b}$ as illustrated in Figure 7. The step length is then calculated according to the following equation:

$$
S L=L_{s s}+L_{d s}
$$

where $L_{s s}$ is the trunk forward displacement during single support from $t_{b}$ to $t_{c}$ as illustrated in Figure 7.

$$
L_{s s}=2 \sqrt{2 l h-h^{2}}
$$

The trunk displacement during double support is estimated to be proportional to the foot size $p$, so it can be estimated using the following equation:

$$
L_{d s}=k \times p
$$




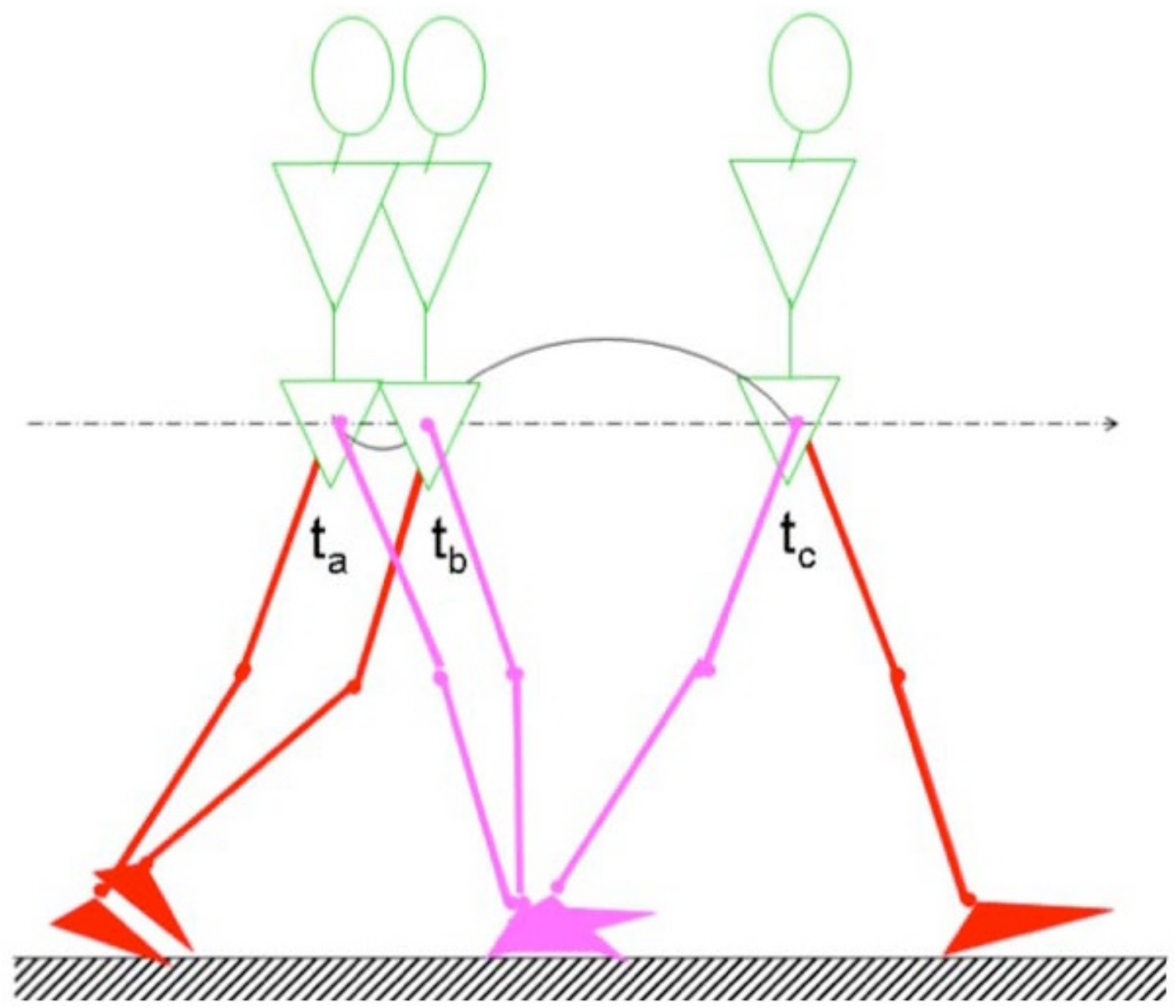

FIG. 7: Step length estimation using a single and double support model [15] 
where $k$ is a constant that has to be calibrated for each person.

\section{Weinberg Equation}

Instead of double integrating the vertical accelerometer data, the following empirical equation was used in [16]:

$$
S L=k \sqrt[4]{a_{\max }-a_{\min }}
$$

where $a_{\max }$ is the maximum vertical acceleration value during a single step, $a_{\min }$ is the minimum vertical acceleration value during the same step and $k$ is constant and need to be calibrated for each user. This method is not subjected to the drift error as it does not require any integration.

A typical calibration method is to instruct each user to walk for a specific distance, then calculate the calibration constant to be used afterwards according to the following equation:

$$
k=\frac{\text { distance }}{\sum_{i=1}^{n} \sqrt[4]{\left(a_{\operatorname{maxi}}-a_{\text {mini }}\right)}}
$$

where $i$ is the step number, $a_{\max i}$ is the maximum vertical acceleration of the $i t h$ step, $a_{\text {mini }}$ is the minimum vertical acceleration of the $i t h$ step and $n$ is the number of steps.

\section{Step Frequency Model}

A linear relation between step length and frequency was found in [5], so the step length was calculated according to the following equation:

$$
S L=a \times f+b
$$

where $f$ is the step frequency, $a$ and $b$ are constants that have to be calibrated for each user. This method is independent of the sensor placement.

\subsection{SUMMARY}

None of the step detection techniques satisfy our system's requirements. All of the algorithms rely on experimental thresholds, while most of them do not distinguish walking from non-bipedal activities. Methods that exploit the walking repetitive pattern will detect any repeated movement withing the walking frequency range 
and they need complex computations. Machine learning techniques can be used to differentiate between walking and other activities, but they require training and complex data labeling, and they can be less accurate than the other methods in detecting the steps.

None of the step length estimation methods satisfied our requirements as well. All of the waist-mounted inertial sensors based algorithms need user calibration. Step length can be estimated from a foot-mounted accelerometer without user calibration but mounting the sensor on the foot is not practical. Gyroscopes are mainly used to transform accelerometer data to world coordinates for step length estimation. The method that use only a gyroscope underestimated the step length and needed experimental calibration. 


\section{CHAPTER 3}

\section{SYSTEM ARCHITECURE}

\subsection{SYSTEM OVERVIEW}

Our main gait analysis system components are depicted in Figure 8. The system is based on two gyroscopes mounted on the lower waist using a belt. Data are collected from the gyroscopes over Bluetooth with a sampling rate of 50 samples/sec by the Data Collection module, then filtered using a Moving Average Filter as a preprocessing for the Step Detection module. If a step is detected, its information is fetched along with the corresponding raw data by the Step Length Estimation module. The Step Length Estimation module can be paired with the vertical acceleration data collected from an accelerometer mounted on the center of the back of the belt near the CoM to auto-calibrate the constant of the Weinberg equation.

In the following sections, we will describe the hardware used, the two sensors placements, and the Data Collection module. The Step Detection module and the data filtering are described in Chapter 4. The Step Length Estimation and the Weinberg Auto-Calibration modules are then described in Chapter 5.

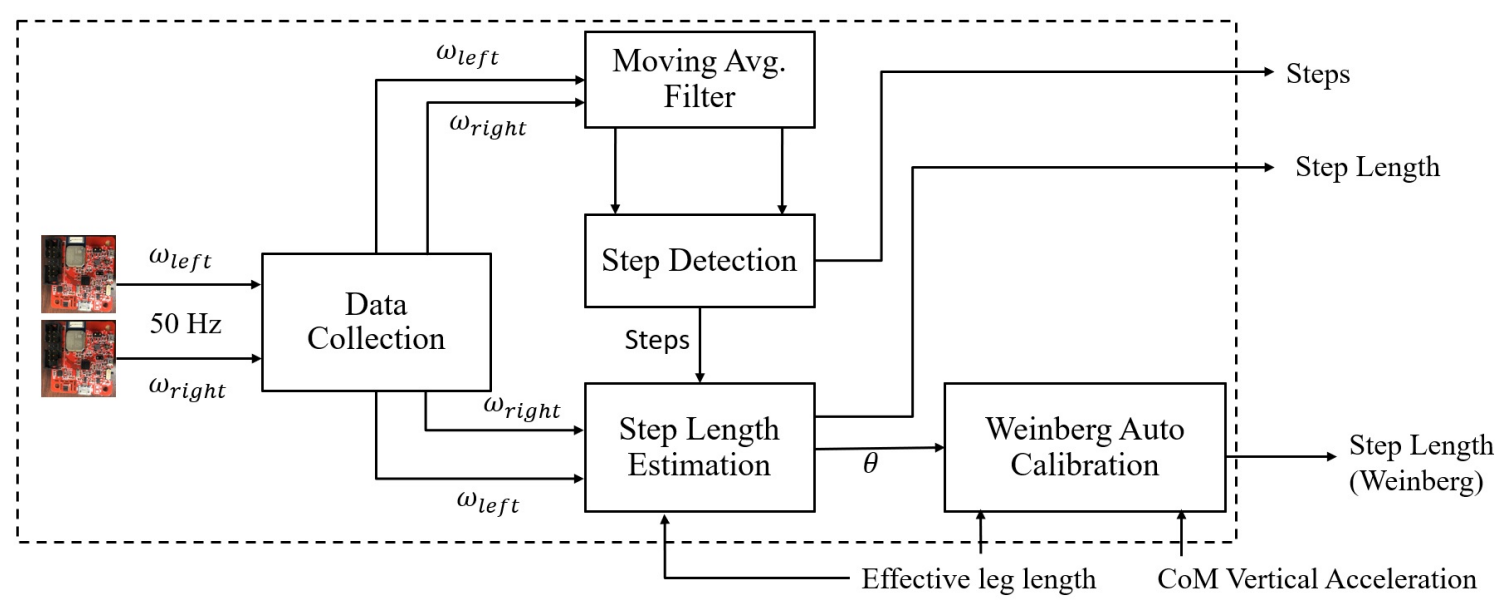

FIG. 8: System Architecture 


\subsection{HARDWARE}

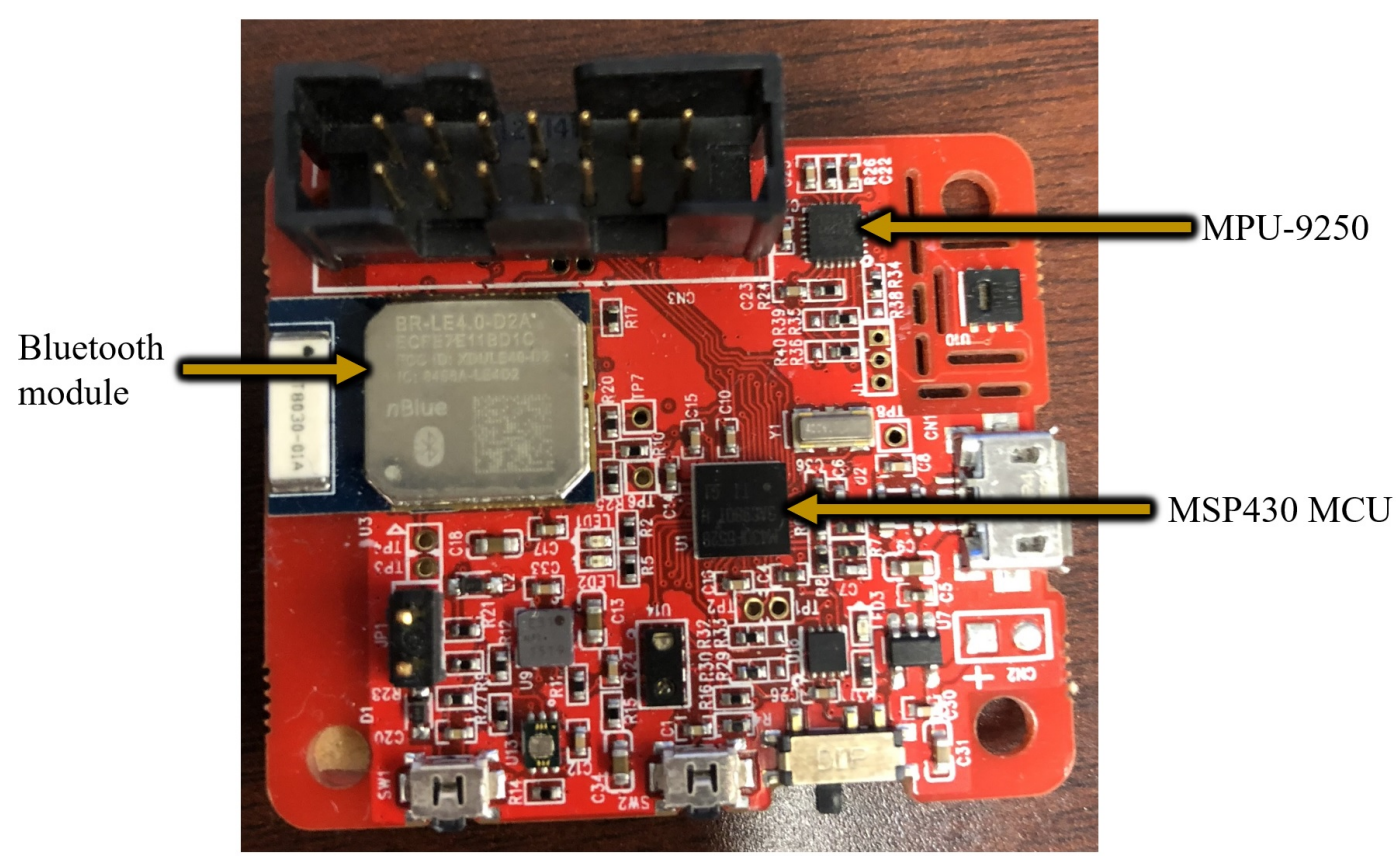

FIG. 9: CA-SDK Board

We use InvenSense CA-SDK board which provides a Motion Processing Unit (MPU-9250), a Bluetooth module for wireless connectivity and an MSP430 Microcontroller unit that provides an interface with the MPU-9250 as illustrated in Figure 9.

The MPU-9250 combines a 3-axis gyroscope, 3-axis accelerometer and a 3-axis magnetometer in a small $3 \times 3 \times 1 \mathrm{~mm}$ quad-flat no-leads $(\mathrm{QFN})$ package. The gyroscope has a programmable full-scale range of $\pm 250, \pm 500, \pm 1000$ and $\pm 2000^{\circ} / \mathrm{sec}$, an operating current of $3.2 \mathrm{~mA}$ and a sleep mode current of $8 \mu \mathrm{A}$. The accelerometer has a programmable full-scale range of $\pm 2 g, \pm 4 g, \pm 8 g$ and $\pm 16 g$, an operating current of $450 \mu \mathrm{A}$ and a sleep mode current of $8 \mu \mathrm{A}$. In our algorithms and experiments, we used the lowest scale range of both of the accelerometer and gyroscope.

\subsection{SENSORS PLACEMENTS}

Mounting the sensors on the waist offers ease of wearability since it can be done by the mean of a belt which can also facilitate the addition of more sensors. The hip can provide useful gait information because it represents the inverted pendulum mass in the stance phase and the point around which the leg rotates in the swing 


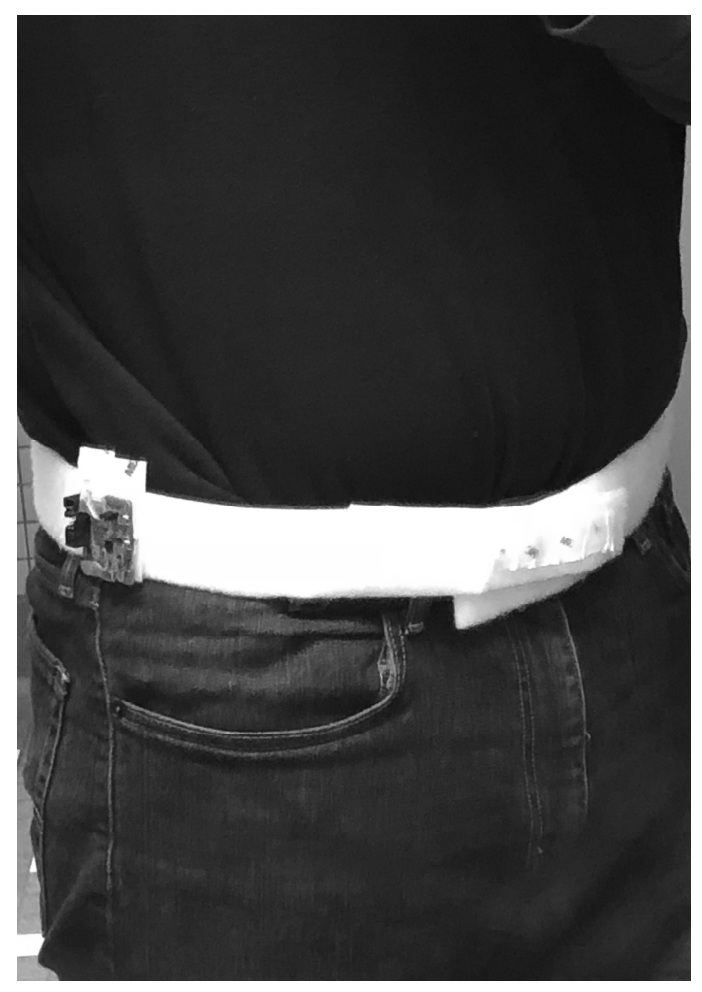

FIG. 10: Right sensor placement

phase. In fact, forward progression cannot happen without the hip flexion [17]. To find the balance between wearability and accuracy, we attach the sensors to a Velcro belt using Velcro patches and position them as close as possible to the upper right and left hip bones. The sensors were positioned with the same orientation of each other.

\subsection{DATA COLLECTION}

We only use the gyroscope axis perpendicular on the plane that divides the body into left and right parts (sagittal plane). The data are collected over Bluetooth and processed by a laptop. The MPU-9250 sampling rate is $50 \mathrm{~Hz}$.

The programmability of the CA-SDK board allows the extraction of the timestamp, but we did not use the board's timestamp for synchronization because of the clock drift. An alternative approach was to timestamp the data at the receiving device (laptop). The synchronization can be easily verified by attaching the boards to each other and move them randomly for a period of time then plot the signals to see whether they match each other or not. 


\section{CHAPTER 4}

\section{STEP DETECTION}

\subsection{PREPROCESSING}

Gyroscope data is filtered before applying the step detection algorithm to reduce noise and improve the accuracy. We used a centered 4-path moving average filter of window size 15. This filter can be implemented with minimal calculations by storing the last $w$ samples, where $w$ is the window size and the summation of the samples. For each new sample $i$, the oldest sample is discarded and subtracted from the sum and the new sample is stored and added to the sum, then the sum is divided by the $w$ to get the filtered $j t h$ sample, where:

$$
j=i-\left\lfloor\frac{w}{2}\right\rfloor
$$

\subsection{STEP DETECTION ALGORITHM}

Our algorithm is based on the detection of the swing phase. We use a peak detection technique where the peak corresponds to the mid-swing, but instead of rejecting false steps using a magnitude threshold, we employ a pattern that exists between the rotations of the two hips, and the repetition of this pattern across subsequent steps.

\section{Hips Rotations}

During the swing phase, the hip rotates in the sagittal plane towards the trunk, this rotation is called hip flexion. On the other hand, during the stance phase, the hip rotates apart from the trunk in the sagittal plane, this is called hip extension. The hip flexion and extension are illustrated in Figure 11.

The swing phase of a foot happens during the single support period where the other foot is in its stance phase, so the angular motion of a hip during its swing phase will be in the opposite direction of the angular motion of the other hip, this 


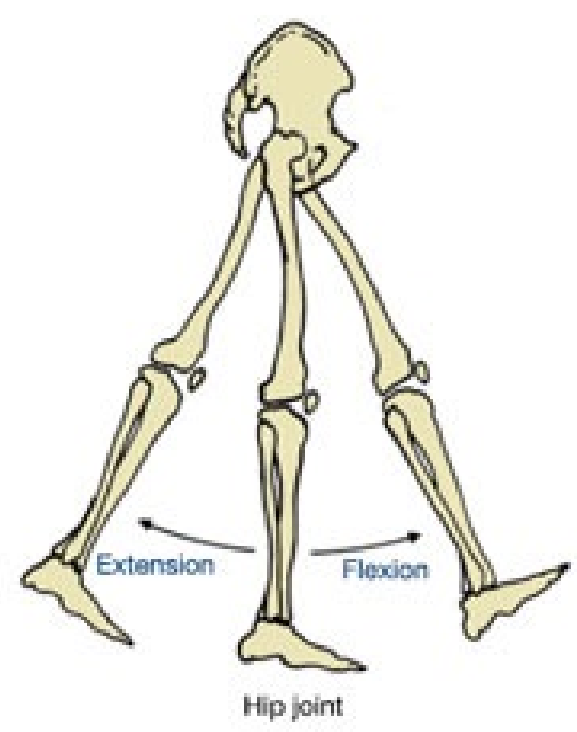

FIG. 11: Hip flexion and extension [28].

pattern of opposite directions can be captured by our gyroscopes' setup. We should expect that during the swing phase of a foot, the data of the corresponding gyroscope should be +ve since the hip is rotating counterclockwise. Also the gyroscope data corresponding to the stance foot should be -ve since the hip is rotating clockwise in the stance phase. After several walking trials, we observed that sometimes the stance leg gyroscope produces +ve fluctuations in the early stance phase, and the pattern of opposite signs always hold starting from the mid-stance point as illustrated in Figure 12. Since the swing leg is modeled as a pendulum, where the mid-stance is the equilibrium position, the mid-stance point can be identified as the point with the maximum angular velocity (+ve peak) of the swing leg.

\section{Pattern Repetition}

During the gait cycle, the swing phase of a foot is succeeded by the swing phase of the other foot. After the last step is taken by a foot, the other foot will swing to reach the last position of the gait. So, to consider that a gyroscope peak corresponds to a gait swing and hence detect a step, it has to be preceded and succeeded by a peak of the other gyroscope, and all of the three peaks have to satisfy the rotation pattern described earlier. This pattern repetition has to happen within the walking 


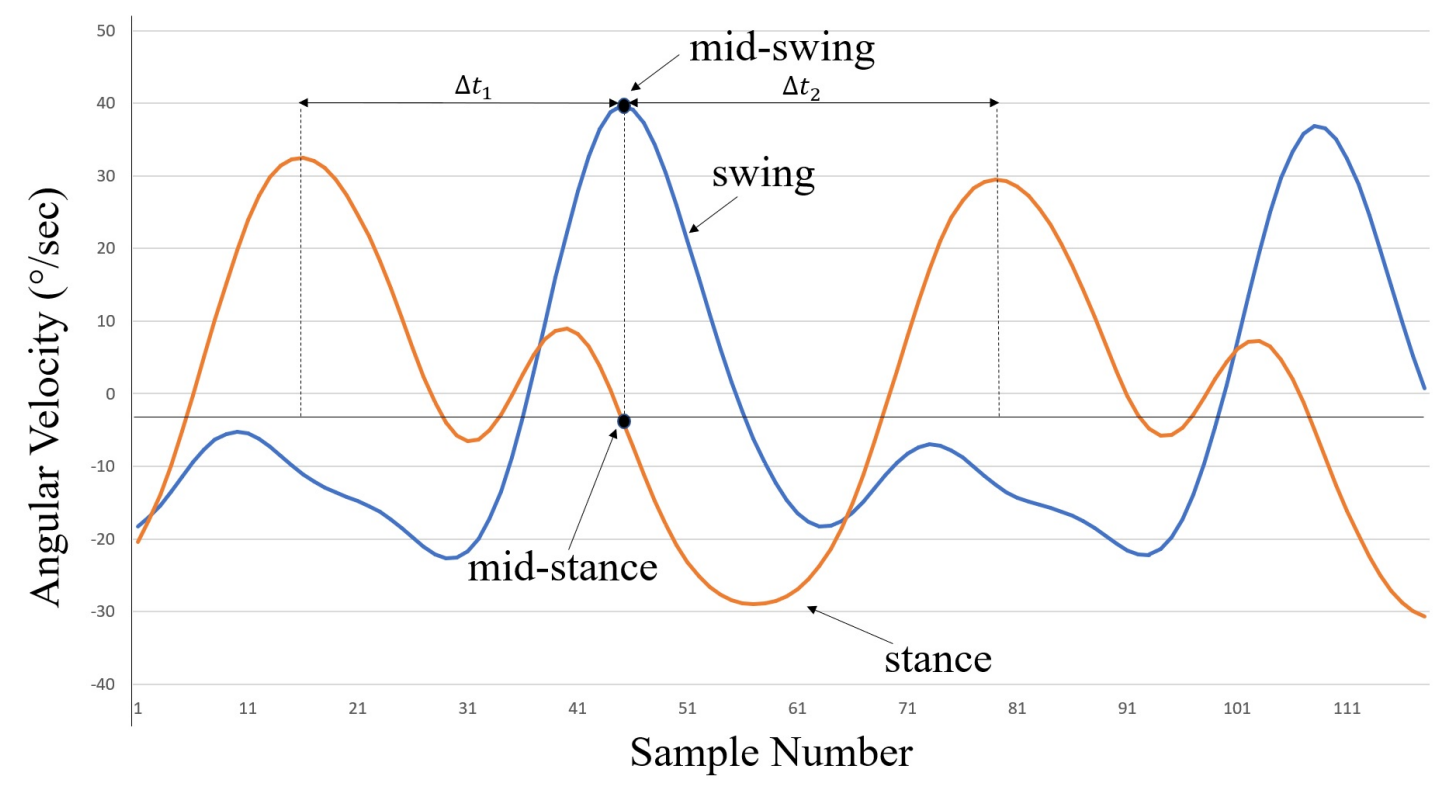

FIG. 12: Plot of two waist mounted gyroscopes filtered data during walking. $\Delta t_{1}$ is the time between the first step and the second step, and $\Delta t_{2}$ is the time between the second step and the third step.

steps expected frequency, so the time between each two subsequent peaks has to be less than a time threshold $\Delta t_{\max }$ and less than another time threshold $\Delta t_{\min }$, where $\Delta t_{\max }$ and $\Delta t_{\min }$ are selected according to [25].

\section{Pseudocode}

The following pseudocode summarize our step detection algorithm, where $\omega_{\text {right }}$ and $\omega_{\text {left }}$ are the filtered readings of the right and left gyroscopes respectively, peak $k_{l e f t}$ and peak $k_{\text {right }}$ are the peaks detected in the left and right gyroscopes signals, peak $k_{l e f t+1}$ is the detected peak of the left gyroscope that succeed peak $k_{l e f t}$, peak $k_{\text {right }+1}$ is the detected peak of the right gyroscope that succeed peak $k_{\text {right }}$, and zeroCrossTime is the time of the signal zero crossing from positive to negative directly after the corresponding peak.

The first and second if-statements check whether a positive value of a sensor overlaps with a peak and its subsequent zero-crossing of the other sensor to reject false peaks. The third and fourth if-statements check if the non-rejected peaks of a sensor is preceded and succeeded by a non-rejected peak of the other sensor within the walking frequency range defined by $\Delta t_{\min }$ and $\Delta t_{\max }$, and the peak to a list of 
valid steps if the conditions are satisfied.

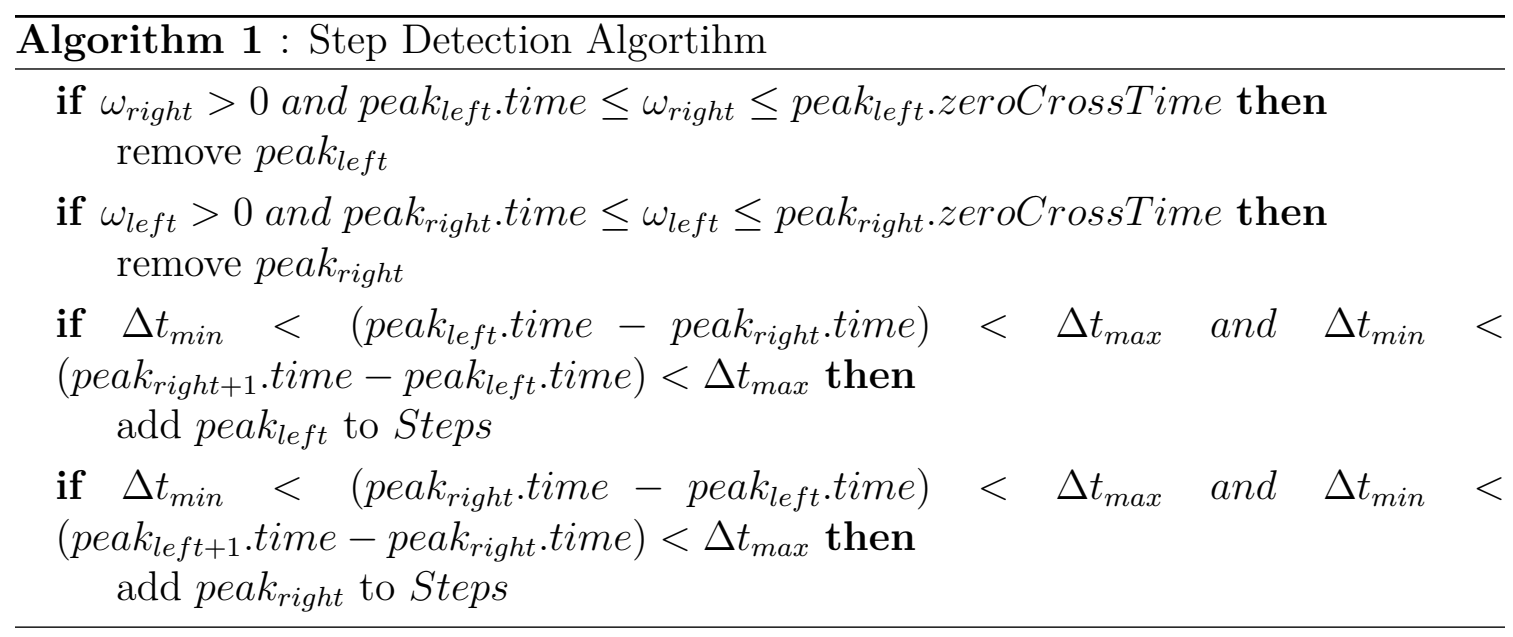




\section{CHAPTER 5}

\section{STEP LENGTH ESTIMATION}

\subsection{STEP LENGTH ESTIMATION METHOD}

We use the gyroscopes to estimate the rotation angle of the hips in stance phase, and we must keep in mind that the gyroscope measures the angular velocity of its rotation around its own axis. Integration of the gyroscope data for the short time period of the stance phase will reduce the drift error significantly and stops it from growing throughout the entire gait. Since the gyroscope signal can experience fluctuation during the early stance, we integrate the gyroscope data during only late stance. The symmetry of the inverted pendulum model allows us to do so, and this also offers the advantage of reducing the integration time which will reduce the drift even more. We use the trapezoidal rule for the gyroscope integration:

$$
\theta=\sum_{i=j+1}^{n+j}\left(t_{i}-t_{i-1}\right) \frac{\omega_{i}+\omega_{i-1}}{2}
$$

where $\theta_{0}$ is the angle at mid-stance, $i$ is the sample number starting from the midstance sample, $t_{i}$ is the timestamp in seconds, $\omega_{i}$ is the gyroscope data value in $\% / s e c$ and $\theta_{i}$ is the calculated angle. Using simple geometry and since the triangle formed by the leg at mid-stance and the same leg at the end of its stance phase in Figure 13 is almost an isosceles triangle, we can use the following derivation to get estimate the step length $S L$ :

$$
\begin{gathered}
\beta=90-\theta \\
\alpha=180-2 \beta \\
\alpha=2 \theta \\
S L=2 l \sin (2 \theta)
\end{gathered}
$$

where $\theta$ is the angle obtained by direct integration of the gyroscope and $l$ is the effective leg length measured from the sensor to the ground.

The error accumulation is low in this method, since we only need to integrate the gyroscope data for a short period of time and we need single integration to get the 


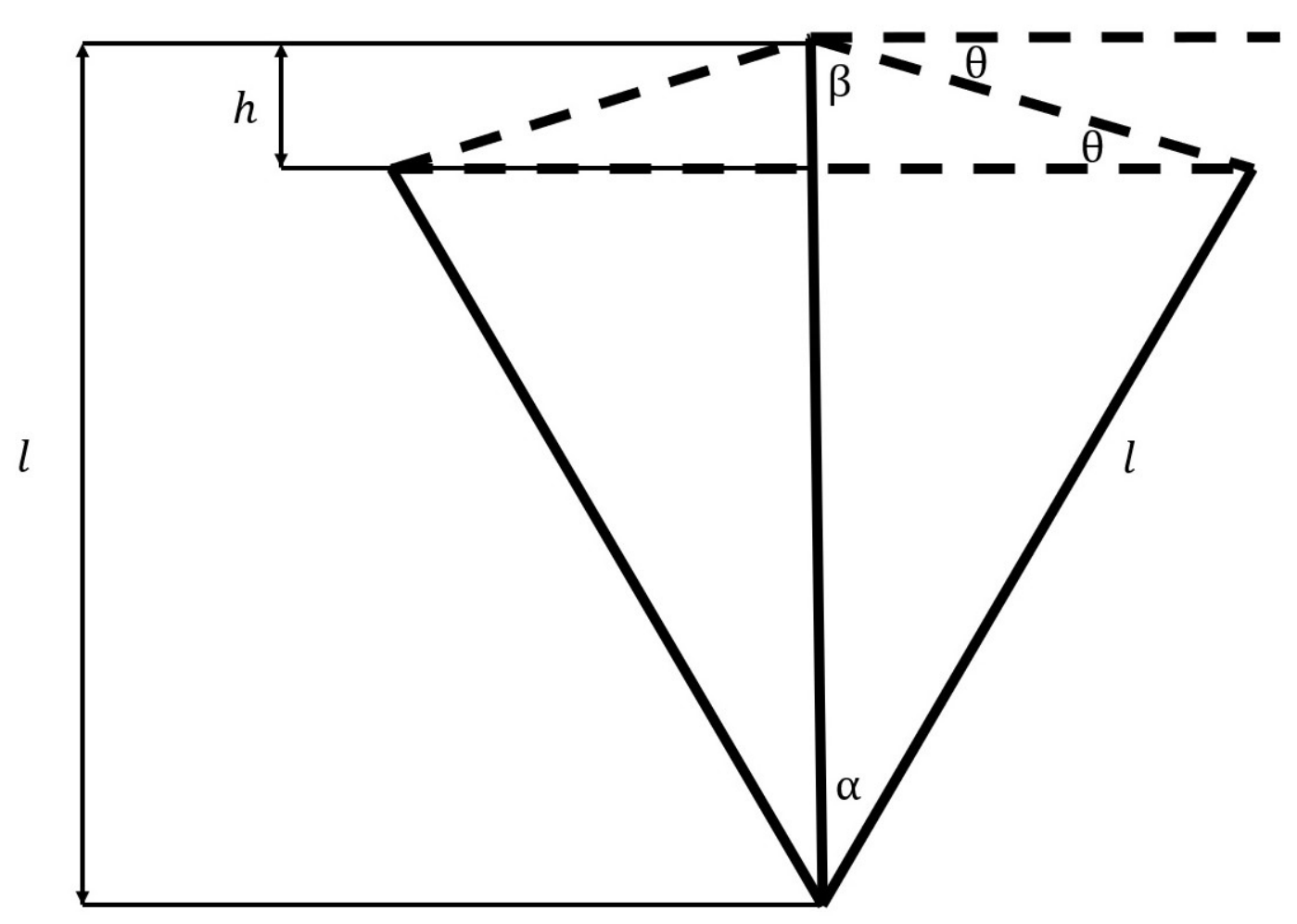

FIG. 13: Walking inverted pendulum model angles

step length unlike the methods based on the double integration of the accelerometer data.

\subsection{WEINBERG METHOD AUTO-CALIBRATION}

The Weinberg method described in Chapter 2 has a high accuracy compared the to other step length estimation methods [13][26], which is confirmed by our experiments. It is not significantly affected by the accelerometer bias since the minimum acceleration is subtracted from the max acceleration leading to the elimination of the bias.

The main disadvantage of the Weinberg method is the need for user calibration which is not user friendly and prone to errors in the user's measurement of the distance. Moreover, we found that the calculated calibration constant does not always span different walking speeds, so if we can find an auto-calibration method, we will increase the accuracy and user-friendliness. The only way for calibration is the walking distance estimation, so we will need another step length estimation method 
if we want to auto-calibrate the Weinberg method. The obvious option is to use a method with more or at least equal accuracy, which we did not find and if it exists we would not need the Weinberg method in the first place. The other option is to use a less accurate method. Step length estimation methods' accuracies vary between different steps. We refer by the method's accuracy to its average accuracy since it is measured for the entire walking distance, so even if a method has a low average accuracy, it may have some high accuracy estimated step lengths. So our problem now is how to detect a high accuracy estimated step length to use for calibration.

By looking at Figure 13, we can conclude that since we can estimate the vertical displacement $h$ using accelerometer integration, that step length can be estimated without knowing the effective leg length according to the following equation:

$$
S L=2 \times \frac{h}{\tan (\theta)}
$$

In fact, the effective leg length $l$ can be estimated given these information using the following equation:

$$
l=\frac{S L}{2 \times \sin (2 \theta)}
$$

To estimate $h$, we attach a CA-SDK chip to the center of the back of the belt to be as close as possible to the CoM, which is the same recommended place for the Weinberg method. We use the method described in [14], which is double integration of the vertical acceleration and estimate $h$ as the difference between the highest and lowest position during each step. But instead of using a high-pass filter to reduce te drift, we use the ZUPT method by restarting the integration at each foot's midstance.

Our experiments showed that the average accuracy of this method was less than $80 \%$, but for some of the steps, $l$ was accurately estimated.

Given the user input of his effective leg length, we first estimate the step length $S L$ using Equation 23, then $l$ using Equation 24. If the accuracy of $l$ is greater than a threshold thresh we use it to estimate a new constant $k_{i}$ using the following equation:

$$
k_{i}=\frac{\sqrt[4]{a_{\operatorname{maxi}}-a_{\operatorname{mini}}}}{S L}
$$

where $i$ is the step number. Then we calculate the Weinberg constant $K$ using a weighted averaging where the weight is the accuracy of $l$.

$$
K=\frac{\sum_{i=1}^{n} k_{i} \times \text { accuracy }_{i}}{\sum_{i=1}^{n} \text { accuracy }_{i}}
$$


where $n$ is the number of steps and accuracy $i$ is the leg length estimation accuracy of step $i$ which will be set to 0 if less than the threshold thresh.

We estimate two calibration constants using the above algorithm, one for slow and one for faster step speeds. A step is considered slow if its duration is less than or equal $0.64 \mathrm{sec}$. This value is calculated by taking the mean of the slow gait mean step frequencies of men and women aging from 10 to 79 years old in [25] and was applicable to the slow walking speed of subjects in our experiments as well. 


\section{CHAPTER 6}

\section{EXPERIMENTS AND RESULTS}

We divided our experiments into 2 categories: walking activity to calculate the step detection and the step length estimation accuracies, and no-walking activities to verify that our step detection algorithm can distinguish walking from other nonbipedal activities. Our ground truth for the step detection was the number of steps counted by an observer, and the ground truth of the step length estimation was done by measuring the overall distance walked using a laser meter and compare it to the overall distance calculated by summing the estimated step lengths.

Two male and one female subjects participated in our experiments. Their ages were 33, 36 and 34 years, their heights were 173, 179 and $169 \mathrm{~cm}$ and their effective leg lengths were $0.95,1.01$ and $0.89 \mathrm{~cm}$ respectively.

\subsection{NO-WALKING ACTIVITIES}

The subjects were instructed to do the following activities: rocking on a rocking chair, twirling on a rotating chair, transitioning between standing and sitting, and jumping in place. Each activity was repeated several consecutive times to ensure that a false step will not be detected due to an activity repetition. Figure 14 shows the two gyroscopes signals of one subject doing the no-walking activities.

None of the activities exhibited the searched pattern between the gyroscopes signals of the two sensors, except for the twirling on a chair activity labeled by A2 in Fig. 14, but this was not detected as a step because the time between the zero crossings was relatively large when compared to the maximum time between two consecutive steps, so the accuracy was $100 \%$ for these experiments.

\subsection{WALKING ACTIVITY}

We asked each one of the subjects to do three experiments, at their slow, regular and fast speed. Each experiment was repeated 5 times to ensure results consistency for a total of 45 experiments runs. The subjects walked in a straight line for different distances ranging from 18 to 32 meters long for each experiment run. Tables 3, 4, 


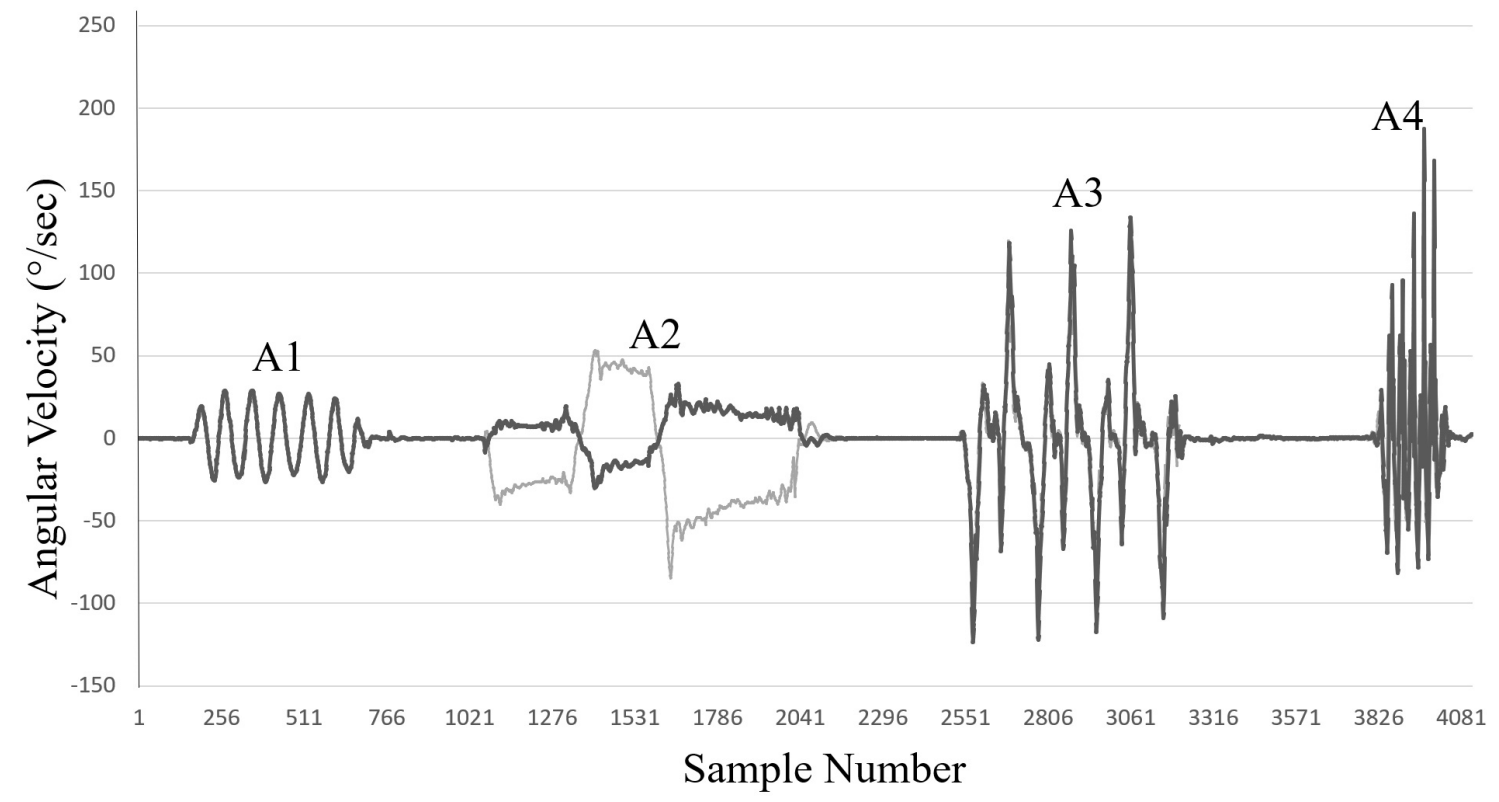

FIG. 14: Plot of two waist mounted gyroscopes filtered data during no-walking activities. A1 corresponds to the activity of rocking on a chair, A2 to twirling on a chair, A3 to transitioning between standing and sitting and A4 jumping in place.

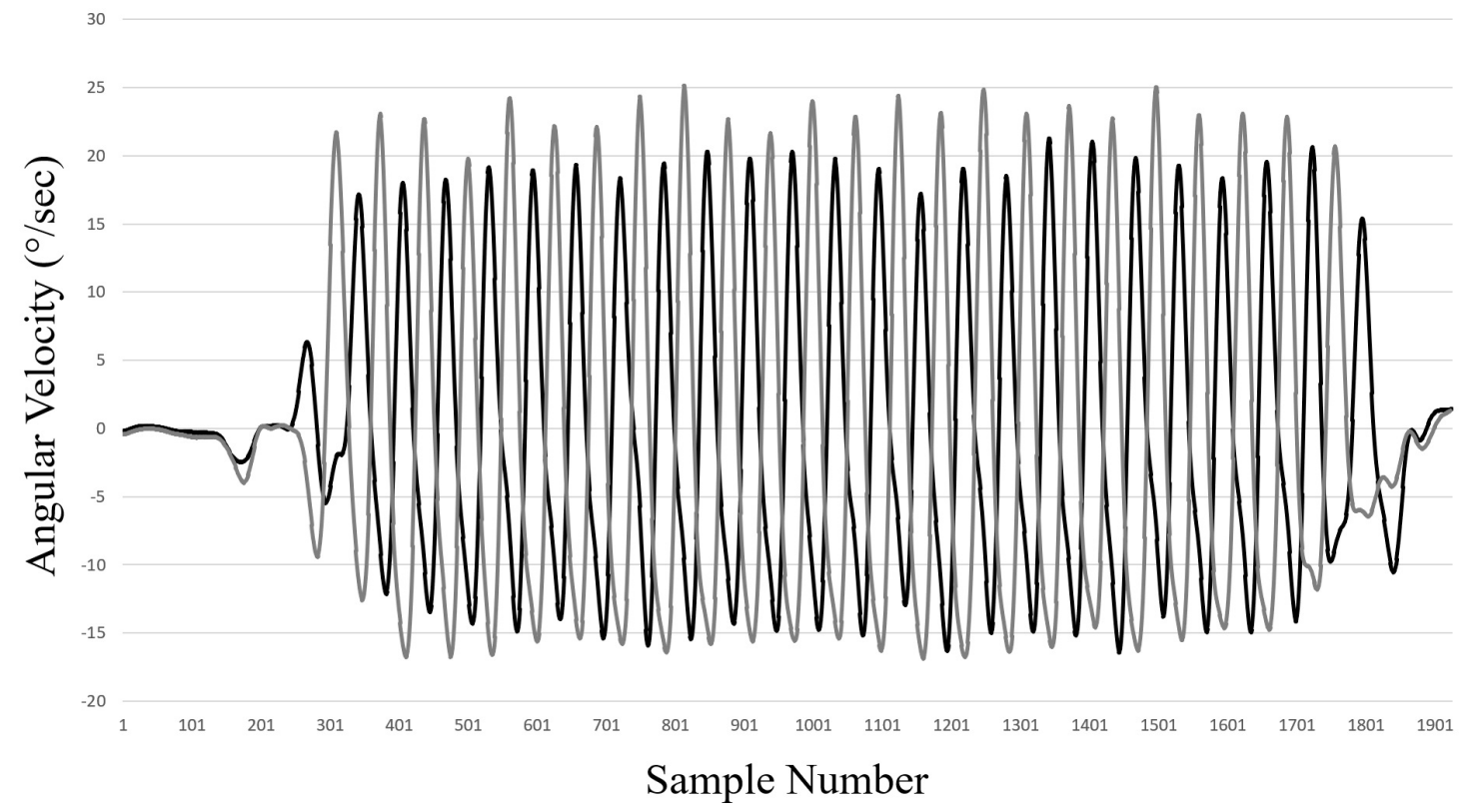

FIG. 15: Plot of two waist mounted gyroscopes filtered data during walking at regular speed. 
and 5 show the gait speed and step frequency of the three subjects for each walking speed.

Figure 15 shows the two gyroscopes signals of one subject walking at his regular speed.

TABLE 3: Slow Gait Speed and Step Frequency

\begin{tabular}{|c|c|c|}
\hline & Speed m/sec & Step Frequency steps/sec \\
\hline Subject1 $(\mathrm{M})$ & 0.769 & 1.4 \\
\hline Subject2 $(\mathrm{M})$ & 0.665 & 1.41 \\
\hline Subject3 $(\mathrm{F})$ & 0.983 & 1.59 \\
\hline
\end{tabular}

TABLE 4: Regular Gait Speed and Step Frequency

\begin{tabular}{|c|c|c|}
\hline & Speed m/sec & Step Frequency steps/sec \\
\hline Subject1 $(\mathrm{M})$ & 1.26 & 1.78 \\
\hline Subject2 $(\mathrm{M})$ & 1.05 & 1.61 \\
\hline Subject3 $(\mathrm{F})$ & 1.47 & 2.11 \\
\hline
\end{tabular}

TABLE 5: Fast Gait Speed and Step Frequency

\begin{tabular}{|c|c|c|}
\hline & Speed m/sec & Step Frequency steps/sec \\
\hline Subject1 $(\mathrm{M})$ & 1.74 & 2 \\
\hline Subject2 $(\mathrm{M})$ & 1.38 & 1.84 \\
\hline Subject3 $(\mathrm{F})$ & 1.6 & 2.21 \\
\hline
\end{tabular}

\subsubsection{STEP DETECTION RESULTS}

The approximate mean accuracy of the step detection was $97.92 \%$ for low walking speed, $99.57 \%$ for regular walking speed and $99.26 \%$ for fast walking speed for a total accuracy of $98.92 \%$. Table 6 shows the sum of the steps taken by the three subjects, and the number of false +ve and false - ve of our step detection algorithm for each walking speed. 
TABLE 6: Step Detection Errors

\begin{tabular}{|c|c|c|c|}
\hline & Steps Count & False $+v e$ & False $-v e$ \\
\hline Slow Walking Speed & 715 & 1 & 11 \\
\hline Regular Walking Speed & 553 & 2 & 1 \\
\hline Fast Walking Speed & 493 & 4 & 0 \\
\hline
\end{tabular}

\subsubsection{STEP LENGTH ESTIMATION RESULTS}

We implemented four methods for step length estimation to compare their accuracies:

1. The first method $M 1$ is the CoM vertical acceleration integration based method described in Chapter 2, we will refer to this method by the vertical displacement method:

$$
S L_{M 1}=K \times 2 \sqrt{2 l h-h^{2}}
$$

We calculated $K$ for each user based on a single walk at regular speed.

2. The second method $M 2$ is our proposed method based on the estimation of the hip angle in sagittal plane:

$$
S L_{M 2}=2 l \sin (2 \theta)
$$

3. The third method M3 is the Weinberg method based on user calibration:

$$
S L_{M 3}=K_{u s e r} \sqrt[4]{\left(a_{\max }-a_{\min }\right)}
$$

4. The fourth method $M 4$ is the Weinberg method based on our new autocalibration technique:

$$
S L_{M 4}=K_{\text {auto }} \sqrt[4]{\left(a_{\max }-a_{\min }\right)}
$$

Figures 16, 17 and 18 show the total estimated distances of all of the experiments as a percentage of the real distances at slow, regular and fast walking speed respectively to show the average estimated step lengths as a percentage of the true step lengths.

Table 7 shows the average accuracy of the four methods at slow, regular and fast walking speeds as well as their overall accuracies. Our proposed method has a 
higher overall accuracy than the vertical displacement method, even though it did not require any constant calibration. Its lowest average accuracy was at fast walking speed and it was higher than the overall lowest average accuracies of the vertical displacement and Weinberg methods. The user calibration constant of one of the users did not span his slow walking speeds for the Weinberg method which decreased the slow walking speed average accuracy significantly. The Weinberg method using auto-calibrated constants has the higher overall accuracy and it is the more stable method across different walking speeds as well. We expect its accuracy to increase even more in practice as the auto-calibration constants is expected to be enhanced with more walking steps.

TABLE 7: Step Length Estimation Accuracies

\begin{tabular}{|c|c|c|c|c|}
\hline & Vertical Disp. & Novel Method & Weinberg & Weinberg Auto \\
\hline Slow Walking Speed & $84.66 \%$ & $92.53 \%$ & $82.25 \%$ & $94.94 \%$ \\
\hline Regular Walking Speed & $92.41 \%$ & $92.34 \%$ & $97.86 \%$ & $95.57 \%$ \\
\hline Fast Walking Speed & $93.68 \%$ & $88.18 \%$ & $96.26 \%$ & $94.02 \%$ \\
\hline Overall & $90.25 \%$ & $91.02 \%$ & $92.12 \%$ & $94.85 \%$ \\
\hline
\end{tabular}

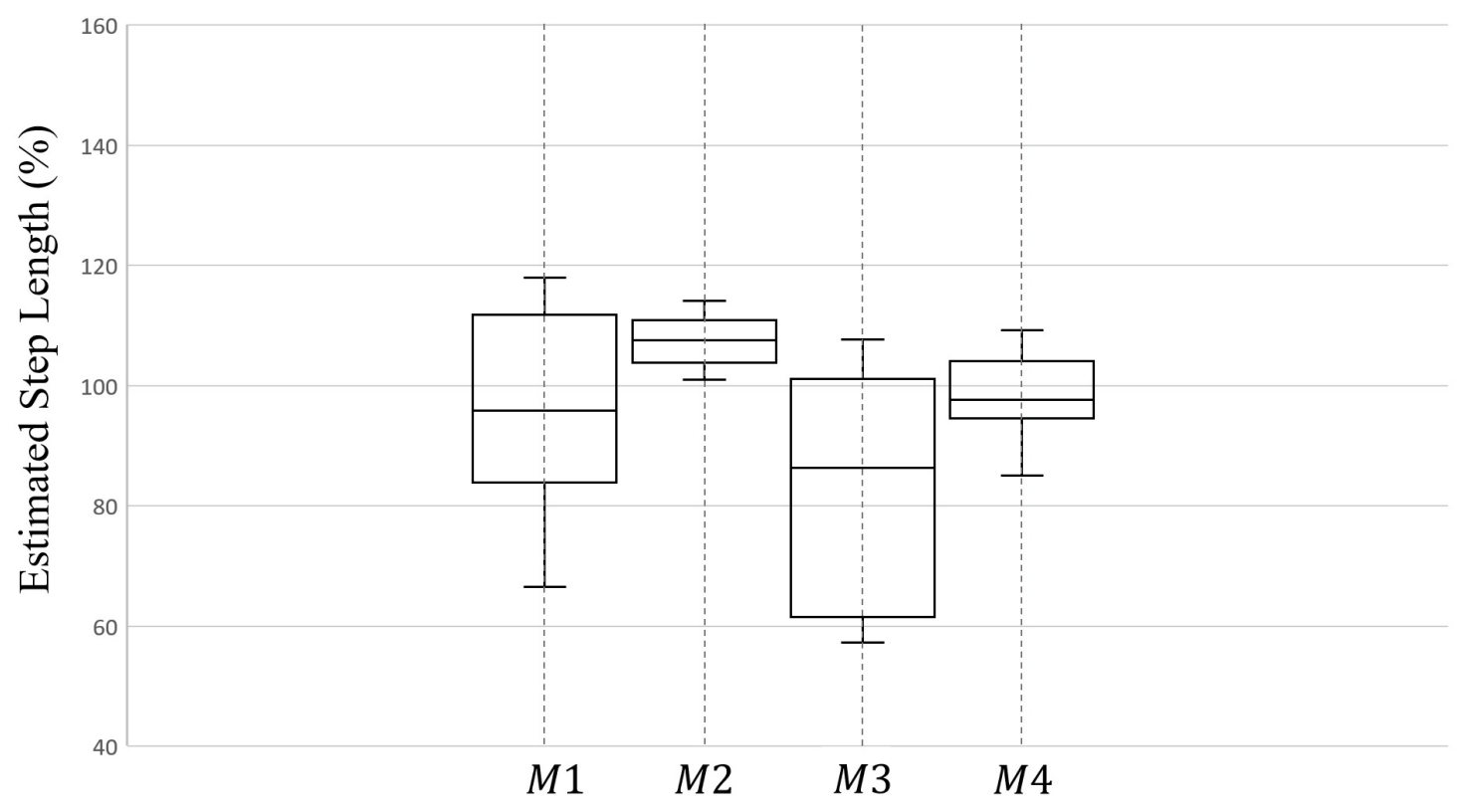

FIG. 16: Plot of the step lengths estimations as a percentage of the real steps lengths at slow walking speed 


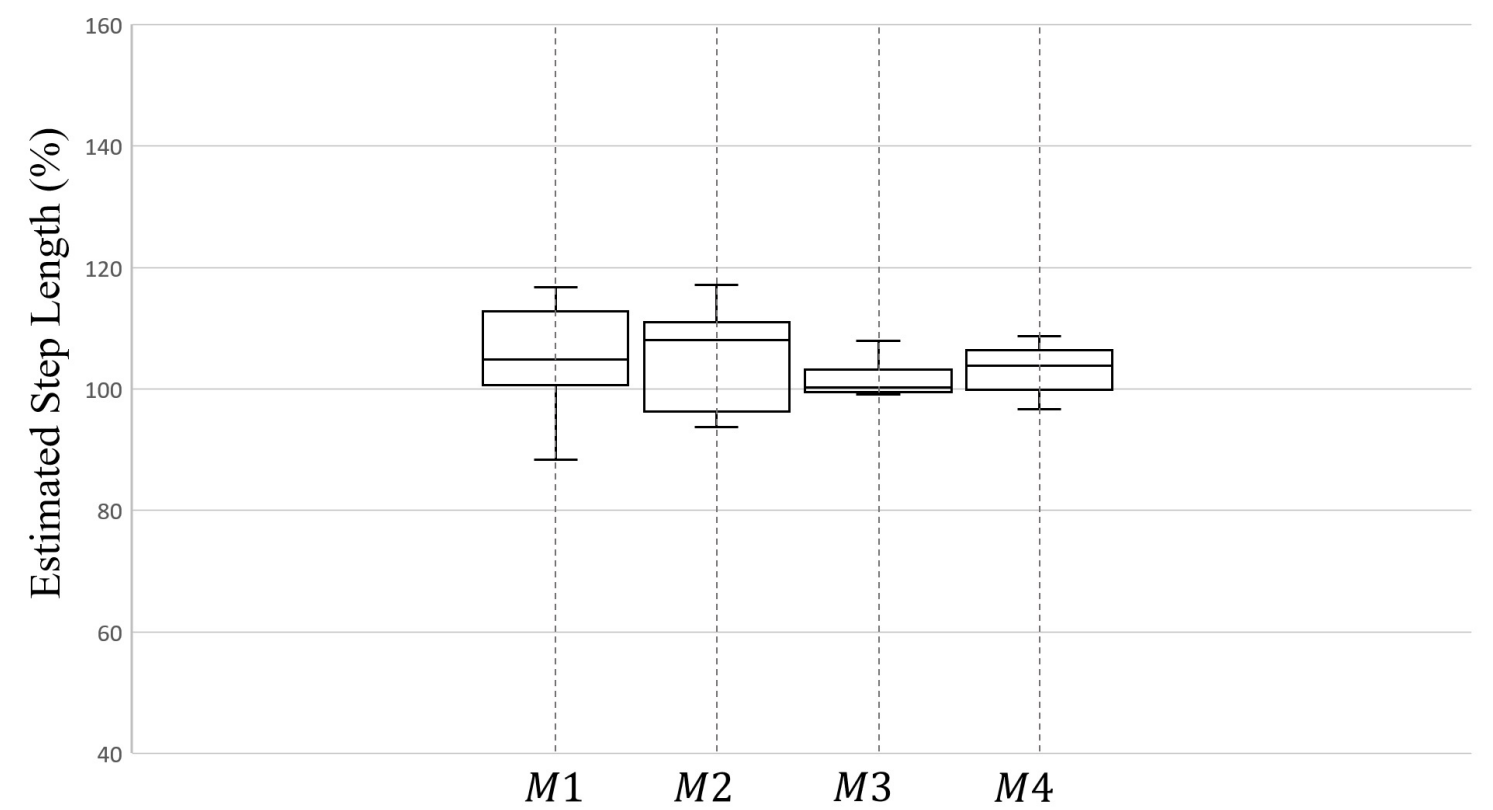

FIG. 17: Plot of the step lengths estimations as a percentage of the real steps lengths at regular walking speed

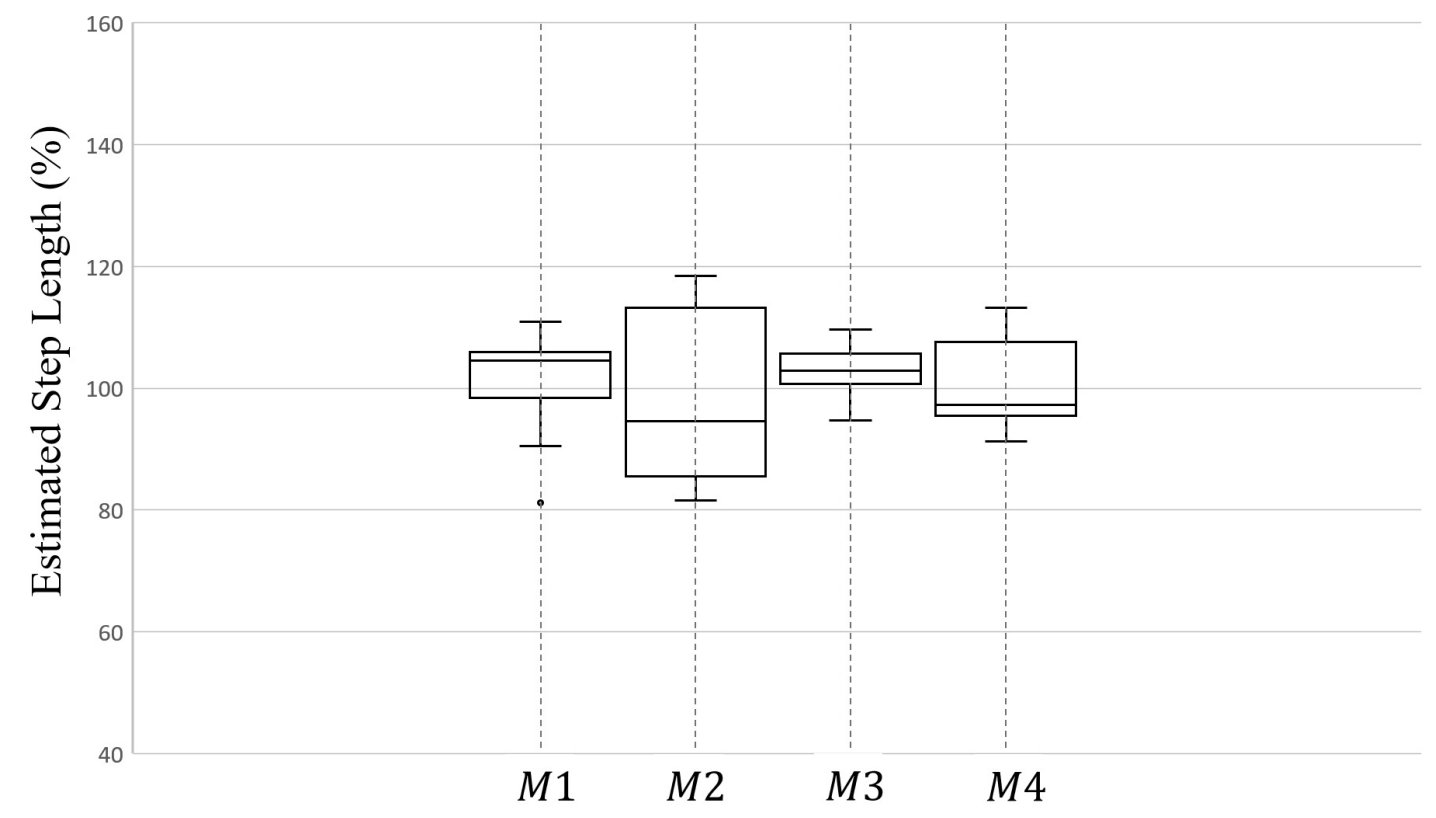

FIG. 18: Plot of the step lengths estimations as a percentage of the real steps lengths at fast walking speed 


\section{CHAPTER 7}

\section{CONCLUSION AND FUTURE WORK}

\subsection{CONCLUSION}

In this thesis, we developed a new method for gait analysis by mounting two gyroscopes, one on each side of the waist. We find that this mounting position is practical and more convenient than other positions such as the feet, shanks or thighs. Using this setup, we were able to accurately detect walking steps and discriminate them from a lot of other non-bipedal activities without using magnitude thresholds or needing for training, which makes our algorithm more robust, easier to implement and more practical than other step detection algorithms. We expect that our step detection method can also be used to accurately detect jogging and running steps, but more experiments are of course needed to evaluate its robustness detecting these activities.

We were also able to estimate the hip rotation angle in the sagittal plane based on the walking inverted pendulum model, which allowed us to estimate the step length using only the user leg length without the need for per user constant calibration unlike all other step length estimation methods that use inertial sensors mounted on the waist. This is also the first gyroscope based step length estimation that does not require further calibration algorithms, and the first waist-mounted gyroscope step length estimation method. We compared our step length estimation method to two other methods that are based on waist-mounted accelerometer. Even though, it does not need calibration constants, our method had a higher accuracy than one of the methods which need a per user constant calibration and a comparable overall accuracy to the other method across different walking speeds.

The estimation of the hip rotation angle allowed us to develop a method to autocalibrate the Weinberg method constant which is one of the most accurate step length estimation methods. We calculate two constants, one for the slow and one for faster walking speeds, and these constants are to be continuously updated while the user is walking. The Weinberg method using the auto-calibrated constants had a higher 
accuracy than all of the other step length estimation methods used in our experiments including the Weinberg method that use user-calibration constant. It was also the most stable method across different walking speeds.

\subsection{FUTURE WORK}

For our future work, we want to be able to estimate the running step length from the waist, so we will need to take into consideration the running additional flight phase. We also want to discriminate walking from other bipedal activities by exploring more unique characteristics of walking. We also want to include more subjects with different ages and physical characteristics in our experiments. Estimating the walking heading is useful, especially in indoor navigation applications, so we want to find a method for heading estimation using multiple gyroscopes mounted on the waist that can achieve a higher accuracy and less computational complexity than traditional methods such as Kalman filtering. 


\section{REFERENCES}

[1] D. Gordon, E. Robertson, https://commons.wikimedia.org/w/index.php? curid=8469181 (accessed July 18, 2018).

[2] Shanahan, Camille J., Frederique Boonstra, L. Eduardo Cofré Lizama, Myrte Strik, Bradford A. Moffat, Fary Khan, Trevor J. Kilpatrick, Anneke Van Der Walt, Mary P. Galea, and Scott C. Kolbe. "Technologies for advanced gait and balance assessments in people with multiple sclerosis." Frontiers in Neurology 8 (2018): 708 .

[3] Cheung, Ada S., Hans Gray, Anthony G. Schache, Rudolf Hoermann, Daryl Lim Joon, Jeffrey D. Zajac, Marcus G. Pandy, and Mathis Grossmann. "Androgen deprivation causes selective deficits in the biomechanical leg muscle function of men during walking: a prospective case-control study." Journal of cachexia, sarcopenia and muscle 8, no. 1 (2017): 102-112.

[4] Rai, Anshul, Krishna Kant Chintalapudi, Venkata N. Padmanabhan, and Rijurekha Sen. "Zee: Zero-effort crowdsourcing for indoor localization." In Proceedings of the 18th annual international conference on Mobile computing and networking, pp. 293-304. ACM, 2012.

[5] Li, Fan, Chunshui Zhao, Guanzhong Ding, Jian Gong, Chenxing Liu, and Feng Zhao. "A reliable and accurate indoor localization method using phone inertial sensors." In Proceedings of the 2012 ACM Conference on Ubiquitous Computing, pp. 421-430. ACM, 2012.

[6] Moore, Steven T., Hamish G. MacDougall, Jean-Michel Gracies, Helen S. Cohen, and William G. Ondo. "Long-term monitoring of gait in Parkinson's disease." Gait \& Posture 26, no. 2 (2007): 200-207.

[7] Gafurov, Davrondzhon, and Einar Snekkenes. "Towards understanding the uniqueness of gait biometric." In Automatic Face \& Gesture Recognition, 2008. FG'08. 8th IEEE International Conference on, pp. 1-8. IEEE, 2008.

[8] Brajdic, Agata, and Robert Harle. "Walk detection and step counting on unconstrained smartphones." In Proceedings of the 2013 ACM international joint conference on Pervasive and ubiquitous computing, pp. 225-234. ACM, 2013. 
[9] Kourogi, Masakatsu, Tomoya Ishikawa, and Takeshi Kurata. "A method of pedestrian dead reckoning using action recognition." In Position Location and Navigation Symposium (PLANS), 2010 IEEE/ION, pp. 85-89. IEEE, 2010.

[10] Fitbit, "How does my Fitbit device calculate my daily activity?" https://help. fitbit.com/articles/en_US/Help_article/1141 (accessed July 1, 2018).

[11] Jayalath, Sampath, Nimsiri Abhayasinghe, and Iain Murray. "A gyroscope based accurate pedometer algorithm." In International Conference on Indoor Positioning and Indoor Navigation, vol. 28, p. 31st. 2013.

[12] Woodman, Oliver J. An introduction to inertial navigation. Technical Report 696. University of Cambridge, Computer Laboratory, 2007.

[13] Jimenez, Antonio R., Fernando Seco, Carlos Prieto, and Jorge Guevara. "A comparison of pedestrian dead-reckoning algorithms using a low-cost MEMS IMU." In Intelligent Signal Processing, 2009. WISP 2009. IEEE International Symposium on, pp. 37-42. IEEE, 2009.

[14] Zijlstra, Wiebren, and At L. Hof. "Assessment of spatio-temporal gait parameters from trunk accelerations during human walking." Gait \& Posture 18, no. 2 (2003): 1-10.

[15] Gonzalez, Rafael C., Diego Alvarez, Antonio M. Lopez, and Juan C. Alvarez. "Modified pendulum model for mean step length estimation." In Engineering in Medicine and Biology Society, 2007. EMBS 2007. 29th Annual International Conference of the IEEE, pp. 1371-1374. IEEE, 2007.

[16] Weinberg, Harvey. "Using the ADXL202 in pedometer and personal navigation applications." Analog Devices AN-602 application note 2, no. 2 (2002): 1-6.

[17] Mochon, Simon, and Thomas A. McMahon. "Ballistic walking." Journal of Biomechanics 13, no. 1 (1980): 49-57.

[18] Muro-De-La-Herran, Alvaro, Begonya Garcia-Zapirain, and Amaia MendezZorrilla. "Gait analysis methods: An overview of wearable and non-wearable systems, highlighting clinical applications." Sensors 14, no. 2 (2014): 3362-3394. 
[19] Annadhorai, Anuradha, Eric Guenterberg, Jaime Barnes, Kruthika Haraga, and Roozbeh Jafari. "Human identification by gait analysis." In Proceedings of the 2nd International Workshop on Systems and Networking Support for Health Care and Assisted Living Environments, p. 11. ACM, 2008.

[20] Woodman, Oliver, and Robert Harle. "Pedestrian localisation for indoor environments." In Proceedings of the 10th international conference on Ubiquitous computing, pp. 114-123. ACM, 2008.

[21] Wang, He, Souvik Sen, Ahmed Elgohary, Moustafa Farid, Moustafa Youssef, and Romit Roy Choudhury. "No need to war-drive: Unsupervised indoor localization." In Proceedings of the 10th international conference on Mobile systems, applications, and services, pp. 197-210. ACM, 2012.

[22] Park, Jaehyun, Yunki Kim, and Jangmyung Lee. "Waist mounted pedestrian dead-reckoning system." In Ubiquitous Robots and Ambient Intelligence (URAI), 2012 9th International Conference on, pp. 335-336. IEEE, 2012.

[23] Feliz Alonso, Raúl, Eduardo Zalama Casanova, and Jaime Gómez GarcíaBermejo. "Pedestrian tracking using inertial sensors." Journal of Physical Agents, vol. 3 (1), pp. 35-42, 2009.

[24] Miyazaki, Shinji. "Long-term unrestrained measurement of stride length and walking velocity utilizing a piezoelectric gyroscope." IEEE Transactions on Biomedical Engineering 44, no. 8 (1997): 753-759.

[25] Oberg, Tommy, Alek Karsznia, and Kurt Oberg. "Basic gait parameters: reference data for normal subjects, 10-79 years of age." Journal of Rehabilitation Research and Development 30, no. 2 (1993): 210.

[26] Alvarez, Diego, Rafael C. González, Antonio López, and Juan C. Alvarez. "Comparison of step length estimators from wearable accelerometer devices." In Encyclopedia of Healthcare Information Systems, pp. 244-250. IGI Global, 2008.

[27] Cavagna, Giovanni A., H. Thys, and A. Zamboni. "The sources of external work in level walking and running." The Journal of Physiology 262, no. 3 (1976): 639-657. 
[28] Nicole Thompson, "Muscles That Move the Leg", https://www.acefitness. org/fitness-certifications/resource-center/exam-preparation-blog/ 3594/muscles-that-move-the-leg, 2017 (accessed July 19, 2018). 


\title{
VITA
}

\author{
Ahmed Nasr \\ Department of Computer Science \\ Old Dominion University \\ Norfolk, VA 23529

\section{EDUCATION}

M.S. in Computer Science, Old Dominion University, USA, 2018.

B.S. in Computer Engineering, Arab Academy For Science and Technology, Egypt 2006.

Typeset using ${ }_{\mathrm{AT}} \mathrm{T}$. 